Rehearsal and Short-Term Retention 1

Running head: REHEARSAL AND SHORT-TERM RETENTION

Rehearsal in serial recall: An unworkable solution to the non-existent problem of decay

\author{
Stephan Lewandowsky \\ University of Bristol and University of Western Australia
}

Klaus Oberauer

University of Zurich

\author{
Stephan Lewandowsky \\ Department of Experimental Psychology \\ University of Bristol \\ 12a Priory Road \\ Bristol BS8 1TU, United Kingdom \\ stephan.lewandowsky@bristol.ac.uk \\ URL: http://www.cogsciwa.com
}




\begin{abstract}
We examine the explanatory roles that have been ascribed to various forms of rehearsal or refreshing in short-term and working memory paradigms, usually in conjunction with the assumption that memories decay over time if they are not rehearsed. Notwithstanding the popularity of the rehearsal notion, there have been few detailed examinations of its underlying mechanisms. We explicitly implemented rehearsal in a decay model and explored its role by simulation in several benchmark paradigms ranging from immediate serial recall to complex span and delayed recall. The results show that articulatory forms of rehearsal often fail to counteract temporal decay. Rapid attentional refreshing performs considerably better, but so far there is scant empirical evidence that people engage in refreshing during short-term memory tasks. Combining articulatory rehearsal and refreshing as two independent maintenance processes running in parallel leads to worse performance than refreshing alone. We conclude that theoretical reliance on articulatory rehearsal as a causative agent in memory may be unwise and that explanatory appeals to rehearsal are insufficient unless buttressed by quantitative modeling.
\end{abstract}


Rehearsal and Short-Term Retention 3

\section{Rehearsal in serial recall: An unworkable solution to the non-existent problem of decay}

Few psychological constructs have been invoked more frequently in memory research than the notion of rehearsal: The overt or covert repetition of to-be-remembered material has taken center stage for several decades of experimentation and theorizing, and the notion of verbal rehearsal is at the core of several classic theories of memory, from the "modal" multi-store model (e.g., Raaijmakers \& Shiffrin, 1981) to the levels of processing framework (F. I. M. Craik \& Lockhart, 1972). Rehearsal is particularly important in theories of memory over the short term, such as Baddeley's working memory model (e.g., Baddeley \& Hitch, 1974; Baddeley, 1986) and related computational instantiations (e.g., Burgess \& Hitch, 1999, 2006; Page \& Norris, 1998). In those models, memories are assumed to decay over time unless they are continually restored through rehearsal. Although there is no logical necessity for rehearsal to be accompanied by decay, all models of short-term or working memory that are known to us and that include rehearsal are also presuming that unrehearsed memories decay inexorably over time (Baddeley, 1986; Barrouillet, Bernardin, \& Camos, 2004; Burgess \& Hitch, 1999; Daily, Lovett, \& Reder, 2001; Kieras, Meyer, Mueller, \& Seymour, 1999; Page \& Norris, 1998; Oberauer \& Lewandowsky, 2011). A tacit assumption in those models is that rehearsal is beneficial - that is, at the very least, rehearsal is seen to offer protection against further forgetting, and at its best, rehearsal is thought to restore memory to its original strength.

This article critically examines the role of rehearsal in memory for serial order over the short term. We first evaluate the empirical claim that rehearsal is beneficial for short-term retention of serial order. Our literature review shows that the evidence is less conclusive than might appear at first glance. Second, we examine the theoretical claim that rehearsal can protect memory traces against decay, by instantiating an empirically-constrained rehearsal regime in a decay model. Our simulations show that, 
contrary to conventional wisdom, in some circumstances additional rehearsal can make memory worse rather than better. We conclude that the explanatory utility of rehearsal in theories of short-term or working memory should not be taken for granted.

At first glance, it may appear unnecessary to re-examine the evidence surrounding rehearsal, given the seemingly well-supported link between rehearsal and memory performance (e.g., Laming, 2008; Rundus, 1971; Tan \& Ward, 2000). For example, using rehearse-out-loud protocols in free recall, Tan and Ward (2000) showed that performance at all serial positions was primarily a function of the recency of an item's last rehearsal, overriding other experimental variables that might affect recall when rehearsal patterns are ignored. Tan and Ward identified the role of rehearsal in free recall as "repeating, reordering, and redistributing the study items throughout the list" (p. 1606), thereby creating multiple avenues for retrieval. Similarly, Laming (e.g., 2008, 2010) has shown that free recall is a function of a memory record that includes traces laid down during rehearsal, in addition to traces formed when items were presented by the experimenter. Generalizing these results to rehearsal in working memory tasks is, however, premature:

Whereas reliance on multiple dispersed copies of the same list items is demonstrably beneficial for free recall, it is inappropriate for serial recall, the principal means by which short-term and working memory are tested. In serial recall, the list must be reproduced in its original order, and re-ordered copies of items made during rehearsal would therefore hinder rather than support accurate recall. We therefore focus our analysis exclusively on serial recall because it offers a particularly challenging environment for rehearsal to exert a memorial benefit.

\section{Rehearsal in Memory for the Short Term: Data}

Because "rehearsal" has been given many different meanings, we distinguish between several psychological processes by which memory can be maintained or 
strengthened. We will consider three forms of rehearsal: articulatory rehearsal, attentional refreshing, and elaborative rehearsal. Elaborative rehearsal involves the integration of material or its enrichment by association with other pre-existing memories (e.g., F. I. M. Craik \& Tulving, 1975). Elaborative rehearsal is known to improve encoding into long-term memory; by contrast, there is little research on the role of elaborative rehearsal in short- term and working memory, and the extant evidence shows that its beneficial effect on memory over the short term is much smaller than on memory over longer times (Loaiza \& McCabe, 2012; Rose, Myerson, Roediger, \& Hale, 2010; Rose \& Craik, 2012). We therefore focus on articulatory rehearsal and attentional refreshing, and defer any further discussion of elaborative rehearsal until after our results have been presented.

The remaining two types of rehearsal maintain or refresh memory traces either through (covert or overt) rote articulation or through non-verbal attentional refreshing. Both mechanisms are frequently invoked in research on short-term or working memory. From here on we use "rehearsal" as a generic term that refers to all forms of memory preservation or restoration. We use rehearsal ${ }_{a r t}$ to refer to rote articulatory rehearsal, and refreshing $_{\text {att }}$ to refer to attentional refreshing. The latter form of rehearsal "... consists of briefly directing attention to a concept or memory, bringing it into conscious awareness" (Ricker \& Cowan, 2010, p. 1356). Rehearsal ${ }_{a r t}$ and refreshing att $_{\text {differ along a number of }}$ dimensions. Unlike rehearsal ${ }_{\text {art }}$, which cannot be applied to material that is neither verbal nor verbalizable (such as unconventional visual characters; Ricker \& Cowan, 2010; Ricker,

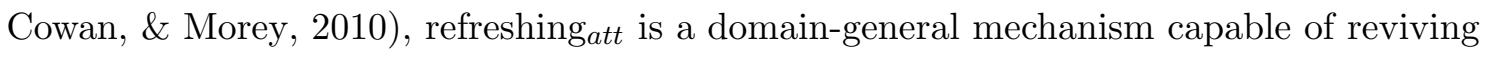
memory traces by simply attending to them (Raye, Johnson, Mitchell, Greene, \& Johnson, 2007). 
Rehearsal and Short-Term Retention 6

\section{Articulatory Rehearsal}

When researchers invoke rehearsal as a maintenance mechanism for short-term retention they usually refer to articulatory rehearsal (rehearsal ${ }_{\text {art }}$ ) of verbal memoranda. The existence of overt or covert articulatory rehearsal is beyond dispute; introspection, everyday observation, and laboratory research all point to the fact that (at least some) people spontaneously recite to-be-remembered information when given a chance to do so. A more pertinent question, therefore, is whether rehearsal ${ }_{\text {art }}$ plays a causal role in mediating memory performance or whether it might be an epiphenomenon, whereby rehearsal is a consequence of a strong memory trace rather than its cause.

The concern that rehearsal may be an epiphenomenon rather than a causal variable is not new (e.g., Watkins \& Peynircioglu, 1982) and rests on the recognition that overt rehearsal, like recall, is a dependent measure. Any association between the two variables thus represents only correlational evidence. To overcome those long-standing concerns, several lines of research have sought evidence for the assumption that rehearsal has a beneficial causal effect on memory.

Suppressing rehearsal. A popular approach has exploited the detrimental effects on memory that arise when rehearsal ${ }_{a r t}$ is prevented. Dating back to the work of J. Brown (1958) and Peterson and Peterson (1959), there is ample evidence that if rehearsal ${ }_{\text {art }}$ is

prevented by some distractor task, such as repeating aloud an irrelevant stimulus, memory performance deteriorates rapidly. Although those effects of articulatory suppression (AS) are strong and pervasive, they suffer from ambiguity of interpretation: On the one hand, the data are compatible with the view that memory inexorably decays when compensatory rehearsal is blocked (e.g., Baddeley, 1986). On that view the data indirectly — via the presumed existence of decay - support a restorative function of rehearsal ${ }_{\text {art }}$. However, the observed impairment to memory could equally result from 
interference between the irrelevant material involved in the suppressor activity and the (non-decaying) memory representations.

The importance of interference in AS was underscored by Gupta and MacWhinney (1995), who decomposed the effects of articulatory suppression into three components. Two of those components were unambiguously ascribed to interference between phonological representations in short-term memory and distracting phonological information: Gupta and MacWhinney identified interference from external sound (i.e., received via the ears) and interference from bone-conductance of sound (i.e., from the generation of speech). Those two components buttress our contention that AS is the result of interference rather than the result of suppressing rehearsal that would otherwise counteract decay. The third component identified by Gupta and MacWhinney was articulatory, and therefore independent of the acoustic attributes of AS. However, the identification of an articulatory component to AS does not necessarily imply that the adverse effects of AS arise from the suppression of rehearsal: Although this was the interpretation advanced by Gupta and MacWhinney within the framework of the phonological loop model, the adverse effects of this component of AS, too, might arise from interference. The work of Gupta and MacWhinney established that people generate a speech plan for the distracting material (i.e., the articulatory component of AS). By the same token, there is evidence that verbal retention in short-term or working memory is also at least partly articulatory in nature (Allen \& Hulme, 2006). The immediate implication of this evidence is that any articulatory representation required for AS - whose existence was established by Gupta and MacWhinney (1995) — would be expected to interfere with a competing articulatory representation of the memoranda. It follows that there are solid empirical grounds to propose that the adverse effects of AS arise not from its suppression of rehearsal but its interference with representations in memory. ${ }^{1}$ 
The interference account of AS differs from the standard explanation because it makes different predictions about the interaction of AS with the effect of time on memory. In contrast to the standard explanation, the interference account predicts that the effect of AS depends on how many different utterances are made, rather than on the duration of AS. On an interference account, the articulatory representations underlying speaking as well as the perceived sound of speaking are encoded into working memory. Hence, every new irrelevant item that is articulated adds interference with the memoranda, but when the same item is continually repeated (such as repeatedly saying "the the the" or "super super super"), further repetitions cause little further decrement in memory performance because the repetitions add no additional information to the content of working memory. By contrast, if different words are articulated (e.g., "Monday, Tuesday, Wednesday, ..."), then those items are all encoded into memory and continue to cause disruption, so that AS leads to more forgetting over longer periods of time. These predictions have been demonstrated through simulations with SOB, a computational model of working memory that incorporates an interference explanation of AS (Lewandowsky, Geiger, \& Oberauer, 2008; Lewandowsky, Geiger, Morrell, \& Oberauer, 2010; Oberauer \& Lewandowsky, 2008; Oberauer, Lewandowsky, Farrell, Jarrold, \& Greaves, 2012). These predictions of the SOB model have received repeated experimental support (e.g., Lewandowsky, Duncan, \& Brown, 2004; McFarlane \& Humphreys, 2012; Oberauer \& Lewandowsky, 2008; Vallar \& Baddeley, 1982). The data from a number of studies are summarized in Figure 1: Whereas Panel A shows the absence of time-based forgetting when rehearsal was prevented by people repeating some distractor (e.g., "the ...the ...the") out loud, Panel B shows that there is a clear effect of extending the duration of a distractor task when the distractors involve variable material (e.g., "Monday, Tuesday, Wednesday, ...").

Taken together, these findings lend greater support to the interference explanation of $\mathrm{AS}$ as incorporated in SOB than to the standard explanation, which assumes that any 
concurrent articulation, regardless of its content, prevents rehearsal. The findings thereby also remove one major empirical motivation for the assumption that rehearsal ${ }_{\text {art }}$ has a causal role in short-term retention.

The word-length effect. Another important presumed piece of evidence for a causal role of rehearsal ${ }_{\text {art }}$ involves the word-length effect (Baddeley, Thomson, \& Buchanan, 1975). Lists of words that take longer to articulate (e.g., "hippopotamus") are recalled worse than lists of words that can be spoken quickly (e.g., "cut"). The word-length effect is thought to arise because lists of long words take longer to rehearse, thus incurring more decay before they can be rehearsed again.

We have shown elsewhere that this interpretation is not unambiguous (Lewandowsky \& Oberauer, 2008). The main reason is that the word-length effect is correlational - it consists of a correlation between two observed variables, the spoken duration of words and the accuracy of recall — and therefore is open to alternative causal explanations. In particular, we argued that the word-length effect might reflect a confound between a word's articulation duration and some other variable that affects memory. Empirical evidence for this possibility was recently provided by Jalbert and colleagues, who showed that word length correlates with the size of a word's neighborhood in the language. When memoranda were equated for neighborhood size, the word-length effect disappeared (Jalbert, Neath, Bireta, \& Surprenant, 2011). We therefore suggest that the word-length effect does not imply a causative role of rehearsal art $_{\text {. }}$

Developmental data. There is much evidence that children's spontaneous engagement in rehearsal ${ }_{a r t}$ increases markedly around age 7 (for a review, see Jarrold \& Tam, 2011). Three "markers" of rehearsal ${ }_{\text {art }}$ begin to emerge between 5 and 9 years; namely, the word-length effect, a phonological similarity effect for visually-presented material, and the relationship between speech-rate and memory span. In the present 
context, we focus on the link between the age-dependent emergence of rehearsal ${ }_{\text {art }}$ and the parallel development of memory span; as Jarrold and Tam noted, "if rehearsal has any beneficial effect on recall at all, then one must predict that the onset of rehearsal confers an increase to memory span in older children" (2011, p. 192).

Contrary to that expectation, a large-scale study of more than 700 children by Alloway, Gathercole, and Pickering (2006) revealed that memory performance increased linearly with age (from 4.5 to 10.5 years) for a set of 12 memory measures, including both verbal and spatial tasks. The same result has been obtained in an equally large study by Gathercole, Pickering, Ambridge, and Wearing (2004). The fact that the shape of the age-performance function did not differ between verbal and spatial tasks is difficult to reconcile with the notion that rehearsal ${ }_{\text {art }}$ helps improve verbal - but not spatial-memories from approximately age 7 onward.

We suggest that although the developmental literature has established the age at which children begin to rehearse verbal memoranda, the available evidence does not compellingly implicate rehearsal as being responsible for better short-term retention. Jarrold and Hall (2012) recently came to much the same conclusion in another review of the available developmental literature.

Rehearsal $l_{\text {art }}$ during encoding in complex span. In the complex-span task memoranda are interleaved with an irrelevant processing task (such as mental arithmetic or a sentence-verification task), and people must recall the memoranda in the correct order immediately after presentation (e.g., Conway et al., 2005; Daneman \& Carpenter, 1980; Lewandowsky, Oberauer, Yang, \& Ecker, 2010). A number of studies have assessed the spontaneous maintenance strategies of participants during a complex-span task using words as memoranda. In the absence of specific instructions, about one quarter to one third of participants report that they repeated memoranda during encoding (i.e., rote rehearsal $_{\text {art }}$; Dunlosky \& Kane, 2007; Turley-Ames \& Whitfield, 2003), another third 
report merely reading the memoranda, whereas the remainder engage in several more sophisticated elaborative strategies (e.g., using imagery; Dunlosky \& Kane, 2007). These studies also investigated whether participants reporting rehearsal ${ }_{\text {art }}$ performed better than participants reporting no rehearsal (i.e., mere reading of the memoranda).

The evidence is ambivalent: On the one hand, Dunlosky and Kane (2007) and Turley-Ames and Whitfield (2003) found little evidence to support the superiority of rehearsal $_{\text {art }}$ over reading. On the other hand, there are some studies which have found better memory among individuals reporting rehearsal ${ }_{\text {art }}$ (Bailey, Dunlosky, \& Kane, 2008; Bailey, Dunlosky, \& Hertzog, 2009; Kaakinen \& Hyönä, 2007). Those studies are however limited by the fact that they relied on self-report to identify rehearsal strategies, which raises the possibility that the reports reflected people's subjective theories about memory rather than their actual strategies - that is, a person may report rehearsal ${ }_{\text {art }}$ because they believe that this is what they would have done, irrespective of whether they actually recollected their activities. Moreover, even if the validity of self-report is taken at face value, this does not resolve the more general problem that these data are once again inherently correlational. They can easily be explained by the plausible assumption that good memory for a list is a prerequisite for rehearsing it.

Experimental manipulations of rehearsal are potentially more diagnostic. In the study by Turley-Ames and Whitfield (2003) participants were explicitly trained in a cumulative-rehearsal strategy (i.e., "as additional words are added to a set, please rehearse aloud, not only the new word, but also other words presented previously in that set," p. 451). In comparison to a control condition that received no guidance about rehearsal, this manipulation raised complex-span performance considerably. However, this effect disappeared when time spent on encoding was controlled (their Experiment 3), suggesting that all other factors being equal, rehearsal ${ }_{a r t}$ had no beneficial effect on performance. 
A micro-analysis of rehearsal. Perhaps the most sophisticated means of examining rehearsal involves recording - and sometimes manipulating - articulatory rehearsals at the level of individual items. This methodology can overcome the correlational problems associated with other evidence. For example, in several of their experiments on free recall, Tan and Ward (2000) presented each participant with a mandatory rehearsal protocol that had been spontaneously generated by a yoked participant under conventional rehearsal instructions. Performance with a prescribed rehearsal schedule largely paralleled that observed with equivalent spontaneous overt rehearsal. This equivalence suggests that the control processes involved in selecting items for rehearsal are not important, and that what matters instead are the additional encoding opportunities afforded by rehearsal. We suggest that this provides evidence for a causal role of rehearsal ${ }_{\text {art }}$.

However, as noted at the outset, the effect of rehearsal in those studies was to repeat, reorder, and redistribute list items (Tan \& Ward, 2000): This can be advantageous for free recall because it generates multiple representations of the same item in different contexts, thereby increasing its chances of recall. By the same token, serial recall would be damaged by reordering and redistributing items in memory. Thus, the findings that support a beneficial role for rehearsal in free recall are unlikely to generalize to immediate serial recall, the most frequently used paradigm for studying short-term memory.

There are only a few micro-analyses of rehearsal ${ }_{\text {art }}$ in serial-recall tasks. Tan and Ward (2008) found a correlation between rehearsal ${ }_{\text {art }}$ during encoding and performance in immediate serial recall. Time permitting, people were found to rehearse cumulatively from the beginning of the list. That is, when presented with the list "A B C D E", people would rehearse "A" after presentation of the first item, followed by "A-B" after the second item, then "A-B-C" after the third item, until the time for rehearsal became insufficient to permit further cumulative recital. At that point, people switched to rehearsing only the last presented item (for a replication of these results see Bhatarah, Ward, Smith, \& Hayes, 
2009). Recall performance was overall strongly correlated with rehearsal; in particular, at early serial positions, correlations between performance and the maximum length of the cumulative rehearsal sequence were as high as .81, with an attenuation of that correlation at later serial positions.

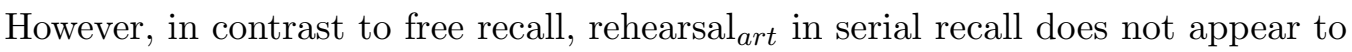
determine the shape of the serial-position curve. Tan and Ward (2008) found the extent of primacy in immediate serial recall to be invariant across different presentation rates, notwithstanding large differences in rehearsal patterns. Specifically, whereas at slow presentation rates rehearsal tended to be cumulative, with faster presentation rates each word tended to be rehearsed only in the interval immediately after its presentation. Because cumulative rehearsal spreads rehearsal of each item across multiple positions, on this scheme early items are rehearsed more often than later list items. In contrast, only rehearsing the last-encoded item assigns equal amounts of rehearsal to all list items. Nonetheless, performance on the first item was only slightly affected by presentation rate, and the primacy effect was nearly equal across presentation rates, even at fast rates when people only rehearsed each item once or twice after its presentation. As Tan and Ward noted, this finding implies that, whereas the primacy effect in free recall can be fully explained by people's rehearsal schedule, in serial recall primacy appears to be largely independent of rehearsal. It follows that rehearsal ${ }_{\text {art }}$ must play different causal roles in free recall and in serial recall.

\section{Attentional Refreshing}

Unlike articulatory rehearsal, which can be measured directly and fairly unambiguously by requesting overt reports from subjects, evidence for refreshing ${ }_{a t t}$ is more indirect and relies on detecting an improvement in memory performance when people are instructed to "think of" an item just presented (Raye et al., 2007), or when the 
time available for presumed refreshing is increased. Whereas earlier work demonstrated a beneficial effect of refreshing for long-term memory, Souza, Rerko, and Oberauer (2015) showed such an effect also for a working-memory task: Instructing participants to "think of" individual objects in a visual array improved their short-term recall of these objects' colors. Vergauwe, Camos, and Barrouillet (2014) present evidence suggesting that people spontaneously engage in refreshing during the retention interval of a working-memory task: Maintenance of verbal or visuo-spatial items in working memory postpones concurrent processing as a function of memory set size, consistent with the idea that people rapidly refresh the entire memory set once before carrying out the processing task.

Barrouillet et al. (2004) pioneered a variant of the complex-span task that has since become a popular tool for investigations of attentional refreshing. In their paradigm, the processing steps in the complex-span task are separated by "free" time, during which there are no overt demands on the participant. When the amount of that "free" time is increased while all other relevant variables remain constant, memory performance improves, which Barrouillet and colleagues ascribe to the increased opportunity for refreshing $_{a t t}$ (e.g., Barrouillet et al., 2004; Barrouillet, Bernardin, Portrat, Vergauwe, \& Camos, 2007). In a series of experiments, Barrouillet, Camos, and colleagues have shown that the ratio between the time taken to process a distractor and the total time available - that is processing time plus free time - is a principal determinant of complex-span performance. This ratio is known as "cognitive load" and ranges from 0 (in which case there are no distractors and the task reduces to simple span) to 1 (in which case distractors are tightly packed together in time, leaving no free time for refreshing). Memory performance turns out to be a linearly decreasing function of cognitive load.

The empirical effect of cognitive load is undisputed, although at least one alternative explanation has been postulated; namely, the active unbinding of interfering distractors from the list context to which they-and the memoranda-were associated. We have 
computationally implemented this mechanism in the SOB-CS model, which successfully reproduces the effect of cognitive load (Oberauer et al., 2012). Independent evidence for the unbinding of irrelevant information has also been adduced (cf. Ecker, Lewandowsky, \& Oberauer, 2013; Ecker, Oberauer, \& Lewandowsky, 2014; Fawcett \& Taylor, 2008).

In summary, although the cognitive-load effect per se is not uniquely diagnostic for a causal effect of refreshing att $_{\text {on memory, evidence for a role of refreshing }}$ att in working memory is beginning to emerge. We therefore assume for the remainder of this analysis that people spontaneously refresh information they hold in working memory. Nonetheless, as we show next, there are some theoretical difficulties associated with the notion of refreshing att $_{\text {. Those difficulties become apparent when the interplay between rehearsal }}$ art and refreshing ${ }_{a t t}$ is considered.

\section{The Interplay of Articulatory Rehearsal and Attentional Refreshing}

An appeal to the existence of multiple types of rehearsal processes has little theoretical force unless it also specifies their interaction. Indeed, recently theorists have

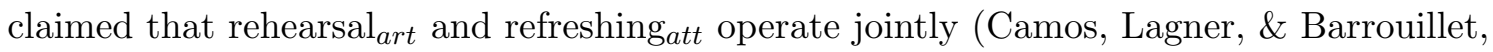
2009). There are several ways in which rehearsal ${ }_{a r t}$ and refreshing att $_{\text {could jointly affect }}$ memory. We consider two options; namely, complete additivity and full sufficiency. We use the label "full sufficiency" because on this option each type of rehearsal on its own can fully restore memory. Figure 2 provides an overview of those two modes of coordination and their implications.

First consider the full-sufficiency scenario (panel B). On this scenario, by definition each type of rehearsal on its own is sufficient to fully restore or preserve memory. The effects of the two types of rehearsal would be under-additive because both of them together do not lead to better memory than each on its own. It follows that if one process is blocked, the other process would be sufficient to counteract adverse consequences of 
that manipulation. For instance, articulatory suppression would prevent rehearsal art but

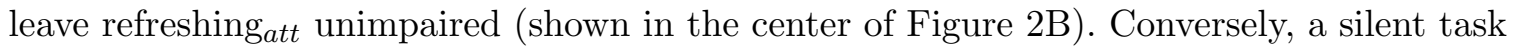
engaging the attentional bottleneck would block refreshing ${ }_{a t t}$ but still permit rehearsal ${ }_{\text {art }}$. According to the full-sufficiency scenario, in both cases no impairment of memory, and no time-based forgetting, should be observed relative to a control condition in which neither rehearsal $_{\text {art }}$ nor refreshing ${ }_{a t t}$ are blocked. This is shown with idealized data in the bottom of Figure 2B. The full-sufficiency scenario therefore entails two strong implications. First, it is incompatible with the popular interpretation of articulatory suppression-namely, that suppression of rehearsal ${ }_{\text {art }}$ permits temporal decay to express itself: If refreshing att $_{\text {is }}$ sufficient by itself to prevent forgetting, then the drastic impairment of memory by articulatory suppression cannot arise from uncompensated decay. Instead, articulatory suppression must exert its effect by other means, for example interference between the to-be-articulated material and the memoranda.

Second, on the full-sufficiency scenario, the cognitive-load effect could not arise from the interplay of decay and refreshing ${ }_{a t t}$ presumed by the theorizing of Barrouillet and colleagues because occupation of the attentional bottleneck by itself would not prevent

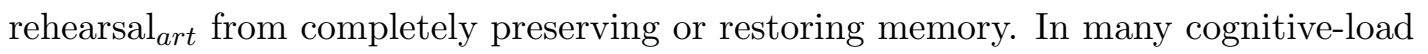
experiments, the distractor task engaging the attentional bottleneck is carried out without articulation, implying that people could rehearse the memoranda, especially when the distractors involve a purely visuo-spatial task (e.g., Barrouillet et al., 2007). It follows that the full-sufficiency scenario is at odds with some of the principal sources of support adduced for the two modes of rehearsal; namely, the effects of articulatory suppression (said to abolish rehearsal ${ }_{\text {art }}$ ) and of cognitive load (said to manipulate the amount of time available for refreshing $a t t)$.

The additivity scenario (Figure 2A) thus appears to be the preferable option. In support, Hudjetz and Oberauer (2007) have shown that a manipulation of cognitive 
load - assumed to affect the opportunity to refresh — and a manipulation of the pacing of articulatory suppression that demonstrably affected the opportunity for rehearsal ${ }_{\text {art }}$ had additive effects on memory. Additivity of manipulations that are assumed to affect

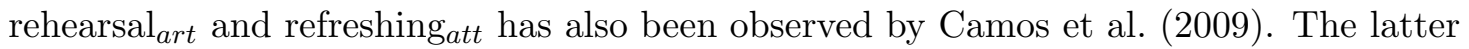
authors interpreted this pattern as showing that attentional and articulatory forms of rehearsal independently and additively provide some of the boost to memory that can occur during the "free" times in a complex-span task.

Thus, the additivity scenario is theoretically more appealing and more consistent with the available evidence than the full-sufficiency scenario. The additivity scenario, however, has important implications of its own, in that it places additional constraints on the interpretation of findings from experiments in which no time-based forgetting is observed when one type of rehearsal but not the other is blocked. Additivity implies that

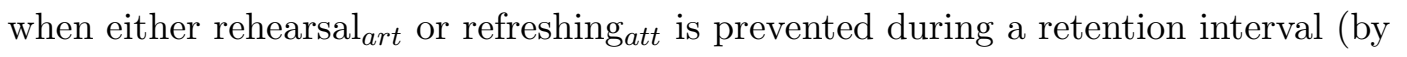
articulatory suppression or by an attention-demanding task, respectively; Oberauer \& Lewandowsky, 2008), time-based decay should manifest itself as forgetting that increases when the period of rehearsal-suppression is extended. This prediction is shown with idealized data in the bottom of Figure 2A. Such time-based forgetting is, however, often absent in the data: Although articulatory suppression severely impairs memory compared to a condition in which it is absent, extending the duration of articulatory suppression does not lead to additional time-based forgetting (e.g., Lewandowsky et al., 2004; Lewandowsky, Oberauer, \& Brown, 2009). Likewise, prolonging the occupation of the attentional bottleneck does not lead to additional forgetting over time (Lewandowsky \& Oberauer, 2009; Oberauer \& Lewandowsky, 2008, 2014). To illustrate with an extreme example, Vallar and Baddeley (1982) showed that preventing rehearsal ${ }_{\text {art }}$ by overt articulation of an irrelevant word ("the") did not lead to any appreciable forgetting during a 15 second delay in a Brown-Peterson paradigm. Similar results have been reported with 
longer lists by Humphreys et al. (2010, 4 digits), Longoni, Richardson, and Aiello (1993, 6 words), and Phaf and Wolters (1993, 6 digits). If rehearsal art $_{\text {formed one of two processes }}$ involved in preserving memory against decay, and their effects were not fully compensatory, then preventing rehearsal ${ }_{\text {art }}$ ought to result in a gradual loss of memory over time. ${ }^{2}$ As we showed earlier (Figure 1A), this time-based forgetting is absent when rehearsal is prevented by people repeating some distractor (e.g., "the ...the ... the") out loud.

One objection to this argument might invoke the time-based resource-sharing model (TBRS) of Barrouillet and colleagues (e.g., Barrouillet et al., 2004). On this view, the number of distractors - and hence the overall duration of the distracting activity - is not expected to have an effect, provided there is time for attentional refreshing in between distractor episodes. That is, the model assumes that memoranda decay during distractor processing, but they are restored by refreshing att $_{\text {(and rehearsal }}$ art in later versions of the theory; Camos et al., 2009) during brief intervals in between distractors or memoranda. In consequence, the model can explain the cognitive load effect discussed earlier, and also why performance frequently remains unaffected by the addition of further distractors in a complex-span task. However, as we have outlined in detail elsewhere (Oberauer \& Lewandowsky, 2014), this prediction can hold only when the amount of memory strength that can be gained by refreshing after each distractor is equal to the loss of strength from decay that occurs during the processing of the distractor. If this condition is not met, for example because there is insufficient time for refreshing att $_{\text {to }}$ to restore the memoranda that have just been weakened by decay, then the addition of further distractors must necessarily reduce performance because decay outpaces restoration. Oberauer and Lewandowsky (2014) derived quantitative predictions from a computational instantiation of TBRS, known as TBRS* (Oberauer \& Lewandowsky, 2011), and confirmed that at higher cognitive loads, increasing the number of distractors was necessarily accompanied by a 
predicted decline in performance. This prediction was disconfirmed by two experiments, which instead showed that performance either remained constant or improved slightly as the number of distractors was increased. Those results affirm the generality of the earlier findings that additional distractors frequently do not impair memory (Humphreys et al., 2010; Longoni et al., 1993; Phaf \& Wolters, 1993; Vallar \& Baddeley, 1982); see Figure 1A. In summary, both scenarios in Figure 2 have implications that are challenged by existing data. Because the additive scenario has been supported by prior theorizing (e.g., Camos et al., 2009), we focus on that option in our simulations below. We do not consider the full-sufficiency scenario further because its predictions appear to be particularly at odds with existing results.

\section{Rehearsal and Memory: Theoretical Mechanisms}

Our survey of relevant research was less conclusive than one might have anticipated: We found little compelling evidence that rehearsal fulfils the role typically ascribed to it in theories of working memory, namely to prevent decay of memory representations. Equally, we found little evidence to rule out that possibility. We now turn to the theoretical question of whether rehearsal can be expected to restore memory representations for serial recall in short-term or working-memory. If rehearsal is to be invoked as an explanatory construct in working-memory research, it must be shown that it actually explains the phenomena it purports to explain. This requires a thorough theoretical investigation of how the presumed mechanisms of rehearsal can be expected to affect memory.

What exactly is rehearsal? Notwithstanding its ubiquity, few explicit specifications of rehearsal ${ }_{a r t}$ exist. For example, some extant models invoke rehearsal without however specifying how it occurs, even if the model's other architectural features are computationally instantiated (e.g., Page \& Norris, 1998). To ascertain the theoretical status of rehearsal as an explanatory construct we need to specify how rehearsal is 
assumed to work. By analogy, Hooke's postulates about gravitational forces did contribute a foundational element to modern physics, but it was Newton's quantification of those postulates into a law of universal gravitation that was required to permit a precise derivation of planetary orbits. ${ }^{3}$

There is considerable agreement that rehearsal requires retrieval from memory; for example, Watkins and Peynircioglu (1982) referred to (articulatory) rehearsal as a "miniature" recall test, and attentional refreshing has likewise been described as requiring “... retrieval from memory by attentional focusing." Barrouillet et al. (2004, p. 84). We therefore conceptualize rehearsal as involving the serial retrieval of memoranda, perhaps into a "focus of attention" (e.g., Oberauer, 2002), followed by the (strengthened) re-encoding of each retrieved item.

We know of only a few existing computational instantiations of rehearsal (Anderson, Bothell, Lebiere, \& Matessa, 1998; Burgess \& Hitch, 1999; Daily et al., 2001; Oberauer \& Lewandowsky, 2008, 2011). Our model is derived from the earlier work by Oberauer and colleagues (we discuss the alternative models once all results have been presented). Oberauer and Lewandowsky (2008) performed a preliminary exploration of the role of rehearsal $_{a r t}$ in serial recall. In addition to instantiating rehearsal $l_{\text {art }}$ in a primacy-gradient model (and finding it wanting), they also presented a positional-decay model that implemented in a generic way the common features of several positional models of serial recall (e.g., G. D. A. Brown, Preece, \& Hulme, 2000; Burgess \& Hitch, 1999; Henson, 1998; Lewandowsky \& Farrell, 2008b; Oberauer \& Lewandowsky, 2011; Oberauer et al., 2012). Perhaps surprisingly, Oberauer and Lewandowsky (2008) found that rehearsal was not always beneficial to performance. 
Rehearsal and Short-Term Retention 21

\section{Simulations}

We implemented rehearsal in the context of a generic model of serial recall in which memory traces decay over time. The model is a scaled-down version of TBRS* that was stripped of as many specific assumptions as possible in order to provide a generic test bed for our exploration of rehearsal. Unlike TBRS*, the present model also included a fully-specified rehearsal $l_{a r t}$ process operating in parallel with refreshing ${ }_{a t t}$. For rehearsal ${ }_{\text {art }}$, the simulations instantiated people's known rehearsal strategies (Tan \& Ward, 2008).

\section{Model and General Method}

Because the TBRS* has been described in full detail elsewhere (Oberauer \& Lewandowsky, 2011), we present a condensed overview of the simplified model used here. The simulation code is available on the first author's personal homepage. The model is a two-layer connectionist network, with one layer representing serial positions and the other layer representing the items (cf. Burgess \& Hitch, 1999, 2006). The two layers are fully interconnected by a matrix of modifiable connection weights. Items are represented in a localist fashion, whereas position representations are distributed, such that positions overlap as a function of their ordinal proximity. Lists are encoded by associating each item to its position by rapid Hebbian learning. During encoding, item-to-position associations are assumed to grow over time towards a constant asymptote, such that the resulting encoding strength of each item is a negatively accelerated function of the duration of encoding. Time permitting, each item is encoded until its encoding strength reaches a criterion, defined as $95 \%$ of the asymptote. As in Oberauer and Lewandowsky (2011), we set the encoding rate $R=6$, a value at which asymptote was reached on average after 500 ms (based on empirical estimates; Jolicoeur \& Dell'Acqua, 1998). Because of random variation in encoding rate (Gaussian, with $\sigma=1$ ), the actual time for encoding each item varied. Figure 3 (Panel a) illustrates the time-dependent encoding of list items. 
One modification from the published version of TBRS* was that we introduced a primacy gradient on the encoding strengths (cf. Lewandowsky \& Murdock, 1989; Page \& Norris, 1998; Farrell \& Lewandowsky, 2004). Introduction of the primacy gradient improved the model's ability to yield realistic serial-position curves. To obtain the primacy gradient, we multiplied the encoding strength for each list item (given by the encoding rate, $R$, and time available for encoding; see Figure 3 ) by $p^{(n-1)}$, where $p=0.9$ is the parameter for the primacy gradient, and $n$ is the item's serial position. The strength of all connection weights between item and position representations decays exponentially over time (irrespective of intervening events) during encoding, retention, and recall. Decay

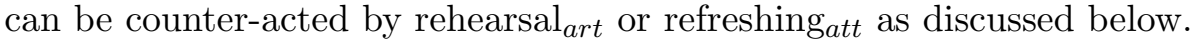

Recall proceeds by cueing each item by its position to regenerate the associated item representation from the weight matrix. Recall duration was assumed to be $0.5 \mathrm{~s}$ per item on average throughout. After recalling an item, response suppression is implemented by (partially) removing its association to the probed position from memory, as detailed in Oberauer and Lewandowsky (2011). Before the addition of rehearsal mechanisms, this model is a bare-bones, generic implementation of those mechanisms that have emerged as common to most successful computational models of serial recall (Farrell \& Lewandowsky, 2004; Hurlstone, Hitch, \& Baddeley, 2013; Lewandowsky \& Farrell, 2008b): Representation of order by item-to-position associations, with position markers overlapping as a function of their ordinal distance, retrieval by competitive cueing, and response suppression.

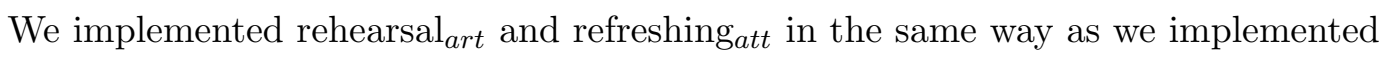
refreshing $_{\text {att }}$ in the original TBRS* model (Oberauer \& Lewandowsky, 2011); the only difference between the two modes of rehearsal is that rehearsal $l_{\text {art }}$ was assumed to take more time. Based on published estimates of the time it takes to rehearse verbal items (Page \& Norris, 1998), we explored rehearsal durations of $250 \mathrm{~ms}$ and $500 \mathrm{~ms}$ per item. 
Those two durations form plausible lower and upper bounds on actual articulation durations. Attentional refreshing was assumed to be more rapid; we used a refreshing rate of $50 \mathrm{~ms}$ per items. This assumption of very rapid refreshing att was needed in our earlier work to account for a number of benchmark data (Oberauer \& Lewandowsky, 2011). Vergauwe and Cowan (2014) recently reported evidence consistent with a refreshing rate of about $40 \mathrm{~ms}$ per item.

As with initial encoding, the duration of re-encoding during rehearsal ${ }_{\text {art }}$ and

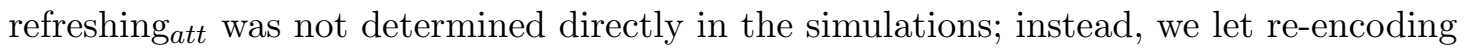
proceed until the amount of additional encoding strength reached a threshold. The threshold was set to a value for which the average duration was the desired duration: For refreshing ${ }_{a t t}$, we set the threshold to $26 \%$ of the asymptote (for a mean duration of $50 \mathrm{~ms}$, see Figure 3 (c)); for rehearsal art $_{\text {we }}$ wed thresholds of $78 \%$ (for a mean duration of $250 \mathrm{~ms}$; see Figure 3 (c)) and of $95 \%$ (for a mean duration of $500 \mathrm{~ms}$ ). Importantly, refreshing $g_{a t t}$ and rehearsal $\mathrm{l}_{a r t}$ were identical in all respects except for their duration. The random variation of encoding rate (as for initial encoding; see above) translated into random variability in the actual durations of refreshing att $_{\text {and }}$ anearsal $\mathrm{l}_{\text {art }}$. Random variability in the durations of encoding, rehearsal, and refreshing are important for the model's behavior because they ensure that the number of items rehearsed, and how far rehearsal progresses into the list, varies from trial to trial.

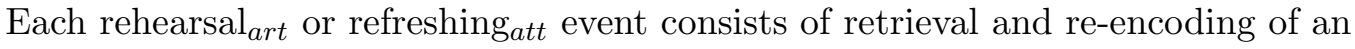
item. Thus, for each rehearsal step, the system selects a serial position among positions already encountered in the current trial; this selection is governed by the rehearsal schedule (to be discussed below). The selected position is used to cue the weight matrix for the associated item, in the same way as for overt recall. The item thus retrieved is re-encoded by associating it to the selected position. Once a new item is presented, rehearsal is interrupted, and to permit encoding, the system steps forward to the position 
following the one at which the preceding item had been encoded, independent of which position was last rehearsed. Note that this implies that the model keeps track of two positions; namely, the one at which the last presented item was encoded, and the one currently to be rehearsed.

To summarize, retrieval and re-encoding during rehearsal occur in largely the same way as retrieval for overt recall and encoding of presented stimuli. One exception is that response suppression is switched off during rehearsal, because response suppression would annihilate the desired effect of re-encoding the item. Moreover, we made the simplifying assumption that re-encoding of the retrieved item starts virtually instantaneously, such that the full duration of a rehearsal step is used for encoding. This is obviously a very generous assumption about the efficiency of rehearsal; a more realistic implementation of rehearsal would assume that some time is needed to retrieve an item and to disambiguate its identity through redintegration (cf. Lewandowsky \& Li, 1994) before re-encoding commences.

All simulations were run for 100 simulated participants, with 100 trials per participant per condition. Table 1 summarizes the experimental paradigms and features of the rehearsal schedules used in all simulations. Table 2 summarizes the values of all parameters for all simulations, with the exception of decay rates which differed within and between simulations and are provided in the text.

\section{Simulation 1}

We begin by examining the effects of rehearsal ${ }_{\text {art }}$ in immediate serial recall by modeling the rehearsal regimes identified by Tan and Ward (2008). Our aim was to reproduce people's observed rehearsal behavior and investigate the consequences that this rehearsal $_{\text {art }}$ has on the model's recall behavior. Tan and Ward (2008) asked participants to rehearse aloud during serial recall with three presentation rates; viz. $1 \mathrm{~s}$ per item, $2.5 \mathrm{~s}$ 
per item, and $5 \mathrm{~s}$ per item. People's overt rehearsals were classified into four different regimes: fixed rehearsal (only rehearsing the last presented item), cumulative forward rehearsal (rehearsing all items up to the last presented one in forward order), partial cumulative rehearsal (rehearsing some but not all of the items presented so far in forward order), and other rehearsal patterns. Because those data are crucial for what follows, we reproduce the main results of Tan and Ward in Figure 4.

The three panels refer to the three presentation speeds (fast, medium, and slow, respectively, from top to bottom) and show that at the fastest presentation rate, people nearly uniformly used the fixed rehearsal schedule (i.e., they rehearse only the last-presented item). At the two slower rates, people tended to use cumulative rehearsal early in the list, and partial cumulative rehearsal in later list positions; at the same time there was still a large proportion of trials in which fixed rehearsal was increasingly used toward the end of the list. This pattern can be explained by assuming that people engage in cumulative forward rehearsal as long as they believe this can be accomplished before being interrupted by presentation of the next item. When the available rehearsal time is judged to be too short for cumulative rehearsal, people instead rehearse only the last presented item. The top panel of Figure 5 shows the serial position curves reported by Tan and Ward (2008).

We implemented the empirically observed rehearsal regimes in the model as follows: After encoding of each item, the model first assessed whether it could rehearse the whole list encoded thus far in the remaining time, by multiplying the number of to-be-rehearsed items (including the just-encoded item) by the mean rehearsal duration. That estimate was used to determine whether or not to engage in cumulative rehearsal:

$$
P=\frac{1}{1+e^{-g \times(d-b)}}
$$


where $d$ refers to the difference between the available time and average expected rehearsal time, $b$ is a bias parameter to represent potential conservatism of people's decision to attempt cumulative rehearsal, and $g$ is a gain parameter representing the consistency of people's decision. For Simulation 1, values of $b=0.4$ and $g=2$ were found to reproduce the observed rehearsal behavior.

If the model decides to rehearse in cumulative forward order, rehearsal commences with the first list item and then moves forward. After each rehearsal step the remaining available time is updated by subtracting the actual rehearsal duration. Because the actual rehearsal durations vary randomly, cumulative rehearsal may or may not run to completion. By contrast, if the initial assessment shows that time will (most likely) not suffice for cumulative rehearsal, the model instead rehearses the last encoded item repeatedly until time runs out. At each decision point, the model engaged in cumulative rehearsal with probability $P$ as determined by Equation 1 and resorted to fixed rehearsal - rehearsing the last-encoded item — with probability $1-P$.

Simulation 1 orthogonally varied three factors: Encoding time per item $(1 \mathrm{~s}, 2.5 \mathrm{~s}$, and $5 \mathrm{~s}$, as in Tan \& Ward, 2008), decay rate (.2 through .8 in steps of .1), and mean rehearsal duration $(0.25 \mathrm{~s}$ vs. $0.5 \mathrm{~s}$ per item). During the presentation of each item, the model first encoded the item into memory (with mean duration $0.5 \mathrm{~s}$ as explained earlier) and then spent the remaining presentation time (i.e., either $0.5 \mathrm{~s}, 2 \mathrm{~s}$, or $4.5 \mathrm{~s}$ ) rehearsing according to the rules just introduced. For comparison we also ran all conditions with rehearsal switched off.

The rehearsal patterns generated by the simulation were classified into the four categories of Tan and Ward (2008); see Figure 6. It is clear from the figure that the simulation with a fast rehearsal rate (250 ms per item) approximately captured the empirically observed distribution of rehearsal categories; compare the left-hand panels of Figure 6 to Figure 4. The simulation captured the general trend in the data of an 
increasing share of cumulative rehearsals with longer presentation durations. However, the simulation yielded more partial cumulative rehearsals and "other" regimes than were observed in the data: those arose because although the model frequently attempted to perform cumulative rehearsal, it also often failed to do so successfully because overall performance was low.

The predicted serial position curves - averaging across all rehearsal schemes - are shown in Figure 7. The figure suggests that under the conditions examined in our simulation, the model cannot simultaneously reproduce the empirically-observed rehearsal schedule and provide realistic serial position curves. None of the predictions in Figure 7 look anything like behavioral serial position curves (i.e., extended primacy with a more confined recency effect). Even the most plausible serial position curves from the simulation, shown in the bottom panel of Figure 5, exhibit massive recency and very little primacy, which is the reverse of the observed pattern (top panel of the same figure). Moreover, the predicted effect of presentation time is the reverse of that shown in the data (compare top and bottom panels of Figure 5). That is, as presentation time increased, performance in the simulation declined whereas it improved in the data.

The source of this problem can be identified by comparing the model's performance with rehearsal (middle and right panels of Figure 7) to its performance without rehearsal (left-hand panels). Because presentation rate is uniformly slower (minimum $1 \mathrm{~s} /$ item) than recall rate $(0.5 \mathrm{~s} /$ item $)$, earlier list items suffer more decay than later list items, thereby generating a strong recency effect. It is apparent that rehearsal is insufficient to compensate for the decay of early list items, and as a consequence, the recency effect still predominates. Note that reducing the extent of decay is insufficient to overcome this problem: Even with the lowest value of the decay parameter (open circles in all panels), the serial position curves are either unrealistically flat (with fast presentation speed) or unrealistically exhibit more recency than primacy (with medium and slow speeds). 
Upon reflection, this outcome is not altogether surprising: If there were decay, and if rehearsal were the only means by which this decay could be counter-acted, then the "fixed" rehearsal regime that is observed at fast presentation rates (top panel of Figure 4) could not possibly give rise to primacy: If only the last item presented is rehearsed at any given stage during list presentation, this sets up a recency gradient because all preceding items will inexorably decay. The empirical fact that extensive primacy is observed even under those circumstances thus presents a challenge to the decay notion.

One objection that might be raised against this conclusion is that it may hinge on a short recall time $(0.5 \mathrm{~s})$ which, by virtue of being faster than the fastest encoding rate, effectively reduces the retention time of later list items compared to earlier ones. If recall duration were $1.0 \mathrm{~s}$ per item instead, thereby equalizing retention time for all items, the serial position curve might be flat. This objection is at odds with data which show that for 6 -item lists, spoken recall of words proceeds at a rate of approximately $0.5 \mathrm{~s}$ per item (Dosher \& Ma, 1998). In addition, Tan and Ward (2008, their Figure 4) showed that extended primacy is observed also for the subset of trials on which people used only fixed rehearsal at the medium and slow presentation rates.

Another way in which a decay model could account for those results is by replacing slow articulatory rehearsal with attentional refreshing. Refreshing ${ }_{a t t}$ is assumed to proceed at a rapid pace, which might provide an opportunity for decay to be counteracted even at short presentation durations.

\section{Simulation 2}

The purpose of Simulation 2 was to examine whether refreshing att $_{\text {can }}$ overcome some of the limitations of conventional rehearsal ${ }_{\text {art }}$ revealed by Simulation 1. Simulation 2 added a fast (50 ms/item) attentional refreshing process to articulatory rehearsal. Because this process escapes direct empirical observation, we had to choose the refreshing regime 
without recourse to data. Given that refreshing ${ }_{a t t}$ is too rapid to be subject to the refined control processes observed for rehearsal ${ }_{\text {art }}$, we assumed that refreshing att $_{\text {would always }}$ commence at the beginning of the list and follow a cumulative forward regime until the next item was presented for study. In line with Camos et al. (2009), the two rehearsal processes were assumed to operate independently.

We implemented the dual-rehearsal model as follows: Once encoding of a memorandum was completed, the model initiated rehearsal ${ }_{a r t}$ and refreshing ${ }_{a t t}$ at the beginning of the list. The simulation proceeded in time steps of $50 \mathrm{~ms}$; at each step, the position of refreshing att $_{\text {was }}$ advanced whereas the position of articulatory rehearsal ${ }_{\text {art }}$ was advanced only every fifth step (i.e., every $250 \mathrm{~ms}$ ). Every progression to a new position was followed by retrieval of an item using the new position as a cue; this item was then re-encoded until the progression to the next position. On steps where only refreshing ${ }_{a t t}$ but not rehearsal $l_{\text {art }}$ progressed, a new item was retrieved for refreshing att but not for rehearsal ${ }_{\text {art }}$. Thus, the model kept track of three positions: the position at which the last item was encoded, the position to be refreshed, and the position to be rehearsed. The model also kept two items in a buffer at any time; viz. the item retrieved for refreshing and the item retrieved for rehearsal $a_{a r t}$.

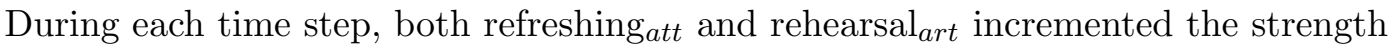
of item-to-position associations by re-encoding the retrieved item for $50 \mathrm{~ms}$. Whereas refreshing att $_{\text {retrieved a new item after every time step, rehearsal }}$ art retrieved a new item only every fifth time step. Because the increment of association strength was exponential, five successive increments of the same association during five successive $50 \mathrm{~ms}$ time steps of rehearsal $\mathrm{l}_{\text {art }}$ were equivalent to one increment for $250 \mathrm{~ms}$; hence each process functioned exactly as it had on its own in the preceding simulations. The cumulative refreshing ${ }_{a t t}$ regime jumped back to the beginning of the list after encoding of each new list item 
whereas the schedule for rehearsal ${ }_{\text {art }}$ was governed by the same mechanism as in Simulation 1. Decay rate was again set to 0.5 .

Paralleling Simulation 1, three different presentation times were used; 1.0 s/item, $2.5 \mathrm{~s} /$ item, and $5 \mathrm{~s} /$ item. The simulation crossed the presence vs. absence of both kinds of rehearsal, yielding 4 cells that explored all possible combinations of the two mechanisms. Figure 8 shows the results. In replication of Simulation 1, a strong recency gradient was observed in the absence of any rehearsal (top-left) or when only rehearsal ${ }_{\text {art }}$ was present (top-right). Refreshing ${ }_{a t t}$ alone overcame the decay-induced recency gradient and yielded plausible, albeit near-ceiling, serial position curves (bottom-left). Finally, when both modes of rehearsal were present, as would be expected under standard serial-recall conditions, the resulting serial-position curves appeared quite realistic (bottom-right). At first glance, the joint operation of both modes of rehearsal thus seems to affirm the ability of the decay model to handle data from serial recall. Upon closer inspection, however, several significant problems remain.

First, as already noted in the context of Simulation 1, the predictions in Figure 8 deviate in an important way from the data: As presentation time increased, performance in the simulation declined whereas Tan and Ward (2008) showed that performance improves with additional time during encoding.

Second, the results in Figure 8 imply that articulatory suppression (AS) should have beneficial effects on recall: AS is commonly understood to prevent rehearsal ${ }_{\text {art }}$ but leave

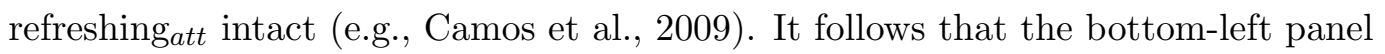
captures the standard AS manipulation. The fact that performance is expected to be better when rehearsal $\mathrm{l}_{\text {art }}$ is blocked than when it is permitted (bottom-right) contradicts a large body of findings to the contrary (e.g., Murray, 1967). In order to explain the pervasive deleterious effects of AS in the data, the model therefore has to invoke some process other than blockage of rehearsal. 
Both of those problems merit detailed analysis which we present after two further simulations involving complex span and delayed recall.

\section{Simulation 3}

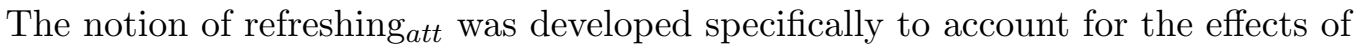
cognitive load in the complex-span paradigm. We simulated complex span with a 6 -item list (presented at the rate of $1 \mathrm{~s} /$ item). Each list item was followed by four distractor operations with a mean duration of $0.5 \mathrm{~s}$. Cognitive load was varied by manipulating the amount of free time, during which refreshing att $_{\text {and/or rehearsal }}$ art was possible, after each item. Free time was either $0.3 \mathrm{~s}$ or $1.0 \mathrm{~s}$; thus generating cognitive loads of $0.5 / 0.8=0.625$, and $0.5 / 1.5=0.33$. Decay rate remained set to 0.5 .

As in Simulation 2, we examined the effects of refreshing ${ }_{a t t}$ and rehearsal ${ }_{\text {art }}$ orthogonally, yielding 4 simulation conditions. We are not aware of any published data that describe people's detailed rehearsal patterns in the complex span task. We therefore instantiated a strict forward cumulative regime for both modes of rehearsal, which proceeded at $50 \mathrm{~ms} /{\text { item }\left(\text { refreshing }_{a t t}\right) \text { and } 250 \mathrm{~ms} / \text { item }\left(\text { rehearsal }_{\text {art }}\right) \text {, respectively. }}^{4}$ Both rehearsals were reset to the beginning of the list after each new item but continued to advance to the next list item after each distractor. We assumed that the distractor task was a non-verbal attention-demanding task. Hence, attentional refreshing was interrupted during distractor processing whereas articulatory rehearsal continued without interruption.

Figure 9 confirms that refreshing att $_{\text {is }}$ essential for a decay model to predict important aspects of complex-span performance. The figure shows that with refreshing att (bottom-left panel), the model produces a cognitive load effect and a serial position curve with extended primacy and a small recency effect, partially consistent with extant data (Lewandowsky, Geiger, et al., 2010). 
Notably, the effects of rehearsal ${ }_{\text {art }}$ again impair the model's ability to account for the data. When rehearsal ${ }_{\text {art }}$ is present on its own (top-right), the model fails to yield a

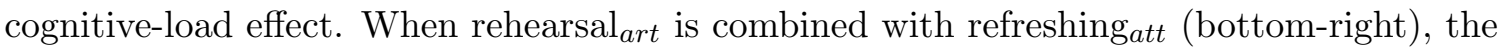
model yields a smaller cognitive-load effect. In addition, as in Simulation 2, adding

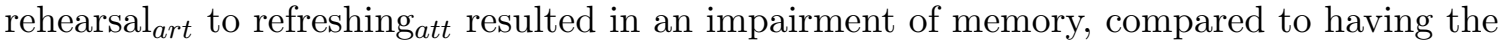
model only refresh. This result resembles Simulation 2 which likewise found that

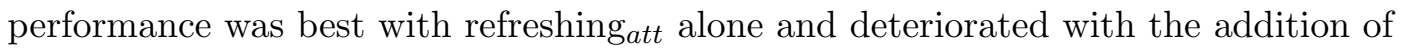
rehearsal $a_{\text {art }}$.

Figure 10 provides another perspective on the results of Simulation 3 that allows a more direct comparison to existing behavioral data. The "refreshing only" and the "both" conditions simulate Experiment 2 of Camos et al. (2009), which involved a parity judgment on digits as distractors. Distractors were presented at a pace of either $0.8 \mathrm{~s}$ (high CL) or $1.5 \mathrm{~s}$ (low CL) per operation, exactly as in our simulation. Subjects made their judgments either by key press or orally. Camos et al. assumed that oral parity judgments suppress rehearsal ${ }_{a r t}$, leaving only refreshing ${ }_{a t t}$ in the free time intervals as maintenance mechanism. Thus, the oral condition corresponds to our refreshing-only condition, whereas the manual condition corresponds to our "both" condition. The "none" and "rehearsal only" conditions in our simulation have no behavioral equivalent in the experiment of Camos et al. (2009). The "rehearsal only" can be thought of as a hypothetical experimental situation in which a (manual) distractor task imposes a cognitive load close to one, so that refreshing ${ }_{a t t}$ is effectively eliminated, but rehearsal ${ }_{\text {art }}$ could still continue. The "none" condition would in addition involve articulatory suppression to prevent rehearsal $a_{\text {art }}$.

Figure 10 shows that despite the fact that rehearsal ${ }_{a r t}$ and refreshing att $_{\text {operated in }}$ parallel and independently in the simulation, their effect on performance in the simulation was far from additive, contrary to the data of Camos et al. (2009). The results imply that 
independent processes need not translate into additive effects of those processes on performance. Conversely, the finding of additive effects in Camos et al. (2009) cannot be taken as evidence for two independent rehearsal processes. ${ }^{5}$

\section{Simulation 4}

We next explored the effects of rehearsal during a retention interval following list presentation. Because we wanted to focus on the effects of rehearsal during retention without any involvement of rehearsal during study, presentation duration was set to $0.5 \mathrm{~s}$ per item. This eliminated any time for rehearsal during list presentation because encoding into memory required the entire duration of each item's presentation. We compared retention intervals of $2 \mathrm{~s}$ and $4 \mathrm{~s}$ during which both modes of rehearsal were again orthogonally present or absent. Based on the assumption that people could not anticipate the end of the unfilled retention interval, both modes of rehearsal proceeded in cumulative forward order.

Figure 11 shows the results in the familiar layout. When no rehearsal was present (top left), recall performance was severely compromised by the additional $2 \mathrm{~s}$ delay in the long retention-interval condition. In contrast to Simulation 1, the serial position curves exhibited some primacy but no recency. The primacy effect arises from the decreasing gradient of activation across the list: Because presentation rate $(0.5 \mathrm{~s} /$ item $)$ in this simulation was equal to recall speed, the retention interval is constant across list positions, and in the absence of rehearsal the serial position curve is therefore entirely determined by the primacy gradient.

Similar to Simulations 2 and 3, the presence of refreshing att $_{\text {on its own improved }}$ performance considerably and nearly eliminated the effect of retention interval (bottom left). Adding rehearsal ${ }_{\text {art }}$ again impaired performance compared to the refreshing-only condition (bottom right). On its own, rehearsal ${ }_{\text {art }}$ was however unable to eliminate the 
effect of retention interval (top right), suggesting that the additional rehearsal opportunity was insufficient to preserve memorial integrity and instead introduced further errors into the list representation.

\section{Discussion of Simulations $1-4$}

The first four simulations converge on the conclusion that rehearsal ${ }_{\text {art }}$ has difficulty serving its often-purported role as a compensatory mechanism for decay. Although Simulation 1 succeeded in reproducing the distribution of rehearsal regimes that were observed by Tan and Ward (2008) at three different presentation rates, the simulation failed to capture memory performance. Across a wide range of parameter values and at all presentation rates, the model mispredicted the serial position curves by producing only recency but no primacy. This failure was a necessary consequence of the "fixed" rehearsal regime that is observed at fast presentation rates (i.e., rehearsal of only the last item presented): When only the last item can be rehearsed, earlier list items inexorably decay, thereby abolishing primacy. Thus, unless one presumes that people's overt rehearsals somehow do not represent their actual, memorially-effective articulatory rehearsals, a decay model cannot simultaneously reproduce the overt rehearsal pattern and recall performance observed by Tan and Ward (2008) with realistic assumptions about the speed of recall.

This problem can be partially overcome by introducing a parallel refreshing ${ }_{a t t}$

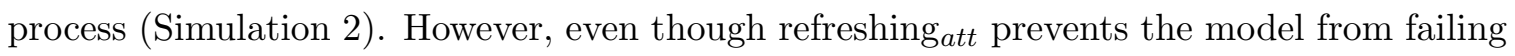
catastrophically, its observed interaction with rehearsal $\mathrm{art}_{\text {rt }}$ points to further problems with articulatory rehearsal: In Simulations 2, 3, and 4, model performance was worse - and more at odds with the data - when rehearsal $l_{\text {art }}$ was operating in addition to refreshing att $_{\text {. }}$

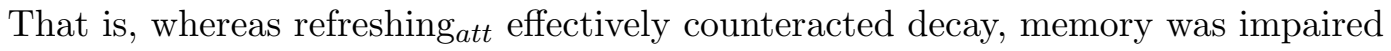
when articulatory rehearsal was added as a second maintenance process running in parallel 
with refreshing - implying that the model expects prevention of articulatory rehearsal (e.g., through articulatory suppression) to have a beneficial effect on performance.

The reasons for the adverse contribution from articulatory rehearsal are illustrated in Table 3, which breaks down performance in Simulation 4 further by focusing on the distribution of the types of "virtual errors" during rehearsal. (As no items are overtly recalled during rehearsal, the errors are virtual rather than actual.) We focused on Simulation 4 because rehearsal occurred only during the retention interval, simplifying analysis because all rehearsal was cumulative.

The table shows that when rehearsal ${ }_{\text {art }}$ is present, a large proportion of rehearsal sequences involves repetition errors. This contrasts markedly with observed recall sequences, among which repetition errors are very infrequent, constituting no more than around $3 \%$ of responses (e.g., Henson, Norris, Page, \& Baddeley, 1996). The excessive number of repetition errors during cumulative rehearsal is a direct consequence of the fact that rehearsal $l_{\text {art }}$, by design, boosts the memory strength of a rehearsed item substantially.

The consequences of this strengthening of memory traces are outlined in Figure 12. Panel A in the figure shows the state of memory after two hypothetical items have been encoded and before rehearsal commences. Rehearsal commences by cueing with the first context markers. This cue retrieves the correct item (panel B), permitting the strengthening of the associations between it and the corresponding context markers (panel C). When the model next attempts to retrieve the second item for rehearsal, the overlap between adjacent position markers implies that the first item is again partially cued (panel D). Because the association of the first item to its position markers has just been strengthened, it may be activated more than the second item when the second item is cued, as is indeed the case in panel D.

In general, when item $n$ has just been rehearsed, there is always a high risk of erroneously retrieving item $n$ again in position $n+1$. This results in encoding a second 
copy of item $n$ in position $n+1$ (not explicitly shown in the figure). Further rehearsal sweeps will usually reinforce this erroneous copy, and at recall, a repetition error occurs. This problem is an inevitable consequence of the fact that rehearsal boosts items one at a time, thereby introducing an imbalance in encoding strength that often overpowers the cueing mechanism. ${ }^{6}$

One implication of this analysis is that if the imbalance in memory strengths created by rehearsal-associated boosts of individual items can be minimized, then the likelihood of repetition errors should decrease and rehearsal might be beneficial after all. This is precisely what is observed with refreshing ${ }_{a t t}$ : The small boosts, accompanied by briefer intervals in between refreshing steps, ensure that refreshing ${ }_{a t t}$ is beneficial to performance because no single list item's strength can ever exceed that of its neighbors to an extent that introduces more errors than the boost in strength prevents. If rehearsal ${ }_{\text {art }}$ makes memory worse when combined with refreshing ${ }_{a t t}$, why does it make memory better when operating alone, in comparison to no rehearsal at all? Switching off both kinds of rehearsal leads to a nearly-complete wipe-out of memory through decay. Relative to that baseline, rehearsal ${ }_{a r t}$ is beneficial because strong boosts of memory strength preserve at least some information about the list, even if fraught with many order errors. Relative to the good performance achieved by refreshing alone, in contrast, adding rehearsal $\mathrm{art}_{\text {adds }}$ little benefit and a substantial risk of introducing errors.

Simulations 1 and 2 also revealed another problem with rehearsal art $_{\text {: Contrary to }}$ the data of Tan and Ward (2008), slower presentation rates - which provided greater opportunity for rehearsal-led to worse performance in the model. We just noted the reasons underlying the deleterious effects of rehearsal, and it is intuitively obvious that those deleterious effects should increase with increasing opportunity of rehearsal. We defer further discussion of the effects of presentation rate to the General Discussion. 
Taken together, Simulations 1 to 4 present us with a conundrum: How can the simulation results be reconciled with the indisputable existence of rehearsal ${ }_{\text {art }}$ in short-term and working memory tasks? Why would people rehearse if their performance were better without it? We suggest that this conundrum is best resolved by discarding with the decay notion and by examining the effects of rehearsal within an alternative, interference-based theory of short-term and working memory. Our final simulations therefore implemented rehearsal in the SOB theory, which has a track record of handling various effects in short-term memory (Farrell \& Lewandowsky, 2002; Lewandowsky \& Farrell, 2008b; Oberauer \& Lewandowsky, 2008) and working memory (Oberauer et al., 2012).

\section{Simulation 5}

SOB-CS assumes that items are represented by vectors of features that are encoded into memory after first being associated to a positional marker via Hebbian learning. All item-context pairings are superimposed onto a common weight matrix. One core feature of SOB-CS, at the heart of much of its predictive power, is that encoding strength is determined by an item's novelty. The more novel an incoming item is relative to what has already been encoded, the more strongly it is encoded into memory. The assumption of novelty-gated encoding has received independent empirical support (Farrell \& Lewandowsky, 2003), and it also serves to explain the observed attributes of AS mentioned earlier: Additional repetition of the same word ("the the the") does not cause further forgetting over time because the novelty of the material is so low that it is not encoded strongly. Alternating material ("Monday, Tuesday, Wednesday"), by contrast, is continually novel and hence encoded more strongly - in consequence, additional utterances during AS cause additional forgetting, as observed in the data (e.g., Lewandowsky et al., 2008). 
Encoding and retrieval processes in SOB are exclusively dependent on events - such as encoding or retrieval of an item-rather than on the passage of time. Thus, forgetting in $\mathrm{SOB}$ results from interference, not temporal decay. To date, SOB has been applied to a wide range of findings without requiring the addition of rehearsal. In particular, even though the model eschews decay or attentional refreshing, SOB-CS has been shown to reproduce the standard cognitive-load effect which had initially stimulated development of the TBRS theory (Barrouillet et al., 2004).

To model the cognitive-load effect in complex-span, Oberauer et al. (2012) assumed that processing a distractor, such as reading a word or carrying out an arithmetic operation, inevitably encodes the distractor into working memory, thereby creating interference with the memoranda. It was furthermore assumed that free time (during which TBRS assumes that refreshing att takes place) is used to "remove" interfering material from memory, by unbinding the distractor representations from its context. The unbinding notion has recently received independent empirical support (Ecker et al., 2013) and is a generalization of the assumption in many models of serial recall (G. D. A. Brown et al., 2000; Burgess \& Hitch, 1999; Farrell \& Lewandowsky, 2002; Page \& Norris, 1998) that once a list item is recalled, it is removed from memory to avoid response perseveration.

We used this latest version of the SOB family to simulate the experiment of Tan and Ward (2008). This required the addition of a rehearsal mechanism into SOB. As in our simulations with the decay model, we initially modeled rehearsal in SOB-CS as pure maintenance rehearsal; that is, retrieval followed by re-encoding of items. Applying SOB-CS to the Tan and Ward experiment required some changes to enable the model to simulate in more detail the pattern of errors people commit; we detail those changes in Appendix A. 
Modeling rehearsal in $S O B-C S$. We modeled rehearsal in SOB-CS in largely the same way as in the decay model: At each step, the current context marker was used to retrieve an item which was then re-encoded into memory by re-associating it with the context marker before adding it to the composite weight matrix. As in the decay model, retrieval during rehearsal differed from overt recall only in that response suppression - that is, removal of a recalled item - was absent. As in Simulation 1, the system tried to rehearse the list in cumulative forward order in the available time between presentation of two successive items. The available time is the inter-item time minus the time for encoding each item (which averages $0.5 \mathrm{~s}$, based on published parameter settings; Oberauer et al., 2012). As in the earlier simulations, each rehearsal step was assumed to take $0.25 \mathrm{~s}$ on average. SOB-CS deterministically switched to a fixed rehearsal regime (i.e., rehearsing only the last-presented item) whenever the remaining time available for rehearsal fell below $0.3 \mathrm{~s}$ before the currently encoded list has been rehearsed completely.

Once SOB-CS switched to fixed rehearsal, it continued with that scheme until the end of the trial. As a consequence, SOB-CS engaged nearly exclusively in fixed rehearsal when presentation rate was fast $(1 \mathrm{~s} /$ item $)$, but it attempted cumulative forward rehearsal during presentation of the first few list items when presentation time was slower (see Figure 13). The model captured the observed rehearsal patterns (Figure 4) reasonably well, although when people perform cumulative rehearsal they do so with greater success than the model.

One feature of the rehearsal protocols of Tan and Ward (2008) is that when participants used a fixed rehearsal schedule, they did not repeat the last-presented list item over and over until the available time ran out. Rather, they spoke the last-presented item once, and occasionally twice, and then fell silent. For instance, at $5 \mathrm{~s} /$ item presentation time, when rehearsal was classified as fixed, the average number of words rehearsed in each inter-item-interval was $1.08 .^{7}$ We captured this behavior in SOB-CS as 
follows: When the model operated in fixed-rehearsal mode, it stopped producing the retrieved item after each repetition with $p=0.8$. When the model stopped overt production, it also stopped re-encoding the rehearsed item. This feature is the only difference between rehearsal in SOB-CS and rehearsal in the decay models: We did not let the decay model stop rehearsing because doing so would mean wasting time during which memoranda further decay but are not rehearsed, thereby putting the model at an even greater disadvantage.

Figure 14 shows serial-position curves for the three presentation time conditions of Tan and Ward (2008), generated from a simulation with rehearsal ${ }_{a} r t$ (solid lines). Unlike with the decay model, in SOB-CS longer inter-item intervals, filled with rehearsal, had no discernible effect on accuracy. Maintenance rehearsal does not benefit memory because in SOB-CS there is no decay that needs to be counteracted by rehearsal. Retrieving and re-encoding an item adds virtually nothing to the memory representation because each item-to-position association has been created at encoding with near-maximum strength, and it maintains this strength until it is being removed from memory owing to response suppression (i.e., after being overtly recalled). Hence, when an item is re-encoded in the same position in which it has been originally encoded, the strength of re-encoding is virtually zero because of novelty-gated encoding: The re-encoded item has very little novelty relative to the existing state of memory.

In principle, re-encoding of items can create interference when a retrieval error occurs during rehearsal: When the wrong item is retrieved, then the re-encoded item mismatches the correct item in the current position, and encoding of a mismatching item is not attenuated by novelty gating, therefore permitting the encoding of a strong interfering signal. Our simulation shows that unlike in the decay model, this theoretical possibility does not translate into a substantial detrimental effect of maintenance rehearsal on accuracy. 
At first glance, the null effect of rehearsal art $_{\text {may }}$ appear to be in conflict with the assumption in SOB-CS that articulatory suppression - which like overt rehearsal entails recitation of verbal material - exerts its effect by interference. There is, however, an important difference between articulatory suppression and overt rehearsal: the speech output generated through rehearsal largely matches the memory list (with the exception of retrieval errors). Owing to novelty-gated encoding, overt rehearsal therefore adds at most minimal interference. By contrast, the speech produced by articulatory suppression differs from the memory list, and because it is therefore novel relative to the contents of memory, creates substantial interference. The dashed line in Figure 14 confirms the interfering effect of articulatory suppression in SOB-CS, modeled by turning off rehearsal and replacing it with the repeated presentation of a constant verbal item every $0.5 \mathrm{~s}$. Items were presented at a rate of one per s, so that two repetitions of the AS stimulus were produced concurrent with each item. In the simulation, encoding of each item was followed by encoding the AS stimulus twice in the same position. AS was assumed to continue uninterrupted, allowing no time for removal.

Rehearsal without decay. The principal message of Simulation 5 is that rehearsal ${ }_{\text {art }}$ does not impair performance within an interference model even though articulatory suppression has an interfering effect as is commonly observed. Unlike within the decay model, additional opportunity for rehearsal ${ }_{\text {art }}$ at slower presentation rates did not reduce performance and did not abolish primacy. Simulation 5 therefore resolved the conundrum raised by the first 4 simulations: If rehearsal is so pervasive and self-evident in serial recall and other working-memory tasks, how can it be so disruptive within a model? The answer is that the problems observed with rehearsal ${ }_{\text {art }}$ are uniquely tied to the assumption of decay and they disappear within the SOB-CS framework.

There is, however, one remaining problem with rehearsal that SOB-CS could not resolve: Whereas Tan and Ward (2008) showed that slower presentation rates improve 
performance, there was virtually no effect of presentation rate in SOB-CS. In SOB-CS, rehearsal is largely inconsequential, because novelty-gated encoding minimizes its causal effect on memory. As a consequence, rehearsal does no damage but also no good. This implies that the beneficial effect of slower presentation rate observed by Tan and Ward (2008) cannot be explained through rehearsal art $_{\text {in }}$ SOB-CS. We sketch out how those effects might be modeled in the General Discussion.

\section{General Discussion}

We begin by clarifying what we did not say in this article. We have not argued against the existence of rehearsal, and we did not reject the idea that rehearsal is an important component of human behavior in short-term memory tasks. The evidence for the existence of articulatory rehearsal as a measurable aspect of behavior during verbal short-term memory tasks is overwhelming and beyond dispute. Instead, what we conclude from the review of the empirical findings is that there is no convincing evidence for a beneficial effect of rehearsal on immediate serial recall. This conclusion leads to our second major point, which pertains to the theoretical utility of rehearsal as an explanatory causal construct.

If one wants to appeal to rehearsal as an explanatory construct, then it has to be demonstrated that assuming rehearsal actually entails the effect to be explained. For instance, if better memory performance in experimental condition A relative to condition $\mathrm{B}$ is to be explained by the assumption that condition A allowed for more rehearsal, then we need to show that the assumption of more rehearsal, in conjunction with other assumptions about the memory system, actually predicts better memory performance in the circumstances of the experiment. We explored this possibility in 4 simulations that instantiated two modes of rehearsal in a generic decay model. The simulations revealed that although rehearsal benefits memory in some circumstances, there are other instances 
in which its benefits are marginal at best. We therefore conclude from the modeling that there is no solid theoretical basis for predicting a uniformly positive effect of rehearsal on memory performance in serial recall.

It is important to highlight that our theoretical conclusions derive not from any failure but from the success of our simulation of rehearsal. Unlike any previous models of rehearsal (e.g., Anderson et al., 1998), our simulations successfully captured the known pattern of human articulatory rehearsal. We consider this successful modeling of rehearsal a prerequisite for any theoretical investigation of what effects on memory to expect from rehearsal. We next recapitulate briefly the ambivalent outcome of that investigation.

The Ambivalent Role of Rehearsal in Short-Term Serial Recall

Articulatory rehearsal. Rehearsal is conventionally understood to be a relatively slow overt or covert articulatory process of around 250-500 ms per item. We found little support for the explanatory value of this modal view of rehearsal ${ }_{a r t}$ : When presentation rate was manipulated, additional opportunities for rehearsal ${ }_{\text {art }}$ were unable to overcome the decay during the added time (Simulations 1 and 2).

Likewise, additional opportunity for rehearsal ${ }_{\text {art }}$ during an unfilled retention interval (Simulation 4) further impaired performance on most list items. The latter results are particularly striking because owing to the fast presentation rates in Simulation 4, no rehearsal took place during encoding - the detrimental effects of additional rehearsal (e.g., top-right panel of Figure 11) thus occurred after the list was encoded to the best of the model's ability. Those results seem at odds with the notion that articulatory rehearsal constitutes a restorative agent in memory.

The reasons underlying this failure became apparent through a detailed analysis of the model's behavior. Contrary to common intuition, rehearsal does not simply add strength to memory representations. When modeled in detail, it becomes apparent that 
rehearsal boosts the strength of items one at a time, and as a consequence, introduces an imbalance of memory strength across items. Consideration of the retrieval mechanisms underlying serial recall (and hence also rehearsal) reveals that these imbalances foster retrieval errors: Items strengthened by rehearsal tend to intrude when the model attempts to retrieve other items to rehearse or recall them (Table 3).

We noted at the outset that rehearsal is most likely beneficial in free-recall experiments, because the re-ordering of items arising from rehearsal does not detrimentally affect free recall performance (e.g., Laming, 2008, 2010). It is therefore notable that within the short-term and working-memory domain, Ward and colleagues have repeatedly highlighted the similarities between free-recall and serial-recall tasks (Grenfell-Essam \& Ward, 2012; Grenfell-Essam, Ward, \& Tan, 2012; Ward, Tan, \& Grenfell-Essam, 2010). At first glance, those results sit uneasily with our main results: If rehearsal assists free recall because re-ordering of items is of no consequence, then how can free recall resemble serial recall in short-term memory if our analysis of the often adverse consequences of rehearsal in immediate serial recall is correct? This slight conundrum can be resolved by considering the fact that even in free recall, people commence output (at least of short lists) with the first item and recall the list (or at least its early part) in forward order. This propensity to commence recall with the first item is enhanced by rehearsal (Grenfell-Essam et al., 2012). It follows that for the short lists considered here, the de facto output order in free recall is not dramatically different from serial recall. Arguably, therefore, people approach a free recall task of short lists as though it were serial recall, and by implication our present analysis would also apply to free recall of short lists - perhaps the advantages of rehearsal in free recall are manifest only when people abandon a forward-serial retrieval strategy, viz. with longer lists.

If, as we suspect, rehearsal ${ }_{\text {art }}$ does not assist serial recall, why do many people engage in it nonetheless? One possibility is, of course, that they act on the subjective 
theory that rehearsal ${ }_{a r t}$ is a helpful strategy for serial recall, even though it is not. Another possibility, which we find theoretically more fruitful, is that people's inclination to (subvocally) repeat verbal lists reflects one of the functions of working memory: The contents of working memory typically serve to guide action, and have a tendency to do so even involuntarily, as has been demonstrated for visual working memory. For instance, visual attention and eye movements in visual-search tasks is derailed towards distractors that resemble a concurrently-held item in visual working memory (Olivers, Meijer, \& Theeuwes, 2006; Soto, Heinke, Humphreys, \& Blanco, 2005). It is entirely plausible that verbal working-memory contents likewise tend to generate corresponding speech acts involuntarily. This assumption is congruent with the ideomotor theory of action control, by which an action is generated by the thought of that action's intended outcome (e.g., Greenwald, 1970; Hommel, 2004).

A further possibility is that engaging rehearsal, even if it sometimes harms memory performance, can be indirectly beneficial by keeping the mind from straying to new thoughts, thereby preventing the worse effects of interference when irrelevant verbal representations are endogenously activated. This possibility finds some support in the amnesia literature. For example, the dense amnesic H.M. whose memory abilities were typically limited to brief retention of a few items, once managed to retain the number 584 for at least 15 minutes, by an elaborate mnemonic scheme that he reported as follows: "It's easy. You just remember 8. You see, 5, 8, and 4 add to 17. You remember 8, subtract it from 17 and it leaves 9 . Divide 9 in half and you get 5 and 4 , and there you are: 584. Easy" (Milner, 1970, p. 37). A short while later, H.M. remembered neither the number nor his own scheme, suggesting that the "rehearsal" did not form a stronger memory but temporarily kept interference at bay. A more formal experimental investigation of the role of interference was recently provided by Della Sala, Cowan, Beschin, and Perini (2005). They examined the role of interference in amnesia and 
conducted a study in which amnesic participants reclined (and occasionally fell asleep) in a dark quiet room for $1 \mathrm{hr}$. After this time, the amnesic patients showed surprisingly intact memory for prose. Della Sala et al. concluded that "the result suggests that long-term memory is encoded in these patients to a greater extent than had been realized but that their memory is highly vulnerable to interference" (p. 435). Perhaps people's recognition of the corrosive effects of interference provides an impetus to engage in rehearsal - even if rehearsal offers little positive memorial benefit.

Attentional refreshing. Our simulations provided more support for the utility of rapid attentional refreshing, as proposed by the TBRS. Although our simulations did not explicitly model an "attentional" process to implement refreshing ${ }_{a t t}$, the simulated speed of this process rules out any involvement of articulation. The speed is however compatible with the notion that retrieval and re-encoding can be performed by an attentional process, assuming that attention can move rapidly from one item to the next. In particular, Simulation 2 showed that attentional refreshing — unlike articulatory rehearsal — enables the decay model to produce reasonable serial position curves (see Figure 8).

Refreshing also succeeded in producing a cognitive-load effect within the decay model. Intriguingly, refreshing att performed best on its own: When it was combined with articulatory rehearsal (Simulations 2-4), performance generally suffered and the model's predictions departed further from the data. In particular, refreshing att $_{\text {erroneously }}$ predicted a negative effect of presentation duration when it was combined with rehearsal $_{\text {art }}$ in Simulation 2 (bottom-right panel of Figure 8).

A further cautionary note arises from the fact that the evidence for the existence of attentional refreshing is largely indirect, being based on interpretation of data in light of one particular theory. Specifically, the cognitive-load effect has been interpreted in support of refreshing ${ }_{a t t}$ based on the verbal predictions of the TBRS theory. Although the TBRS theory does predict the cognitive load effect when instantiated in a computational 
model (Oberauer \& Lewandowsky, 2011), the link between refreshing and cognitive load has been weakened by the existence of an alternative computational model, SOB-CS, that can reproduce the same effect (and numerous associated phenomena) without any refreshing (Oberauer et al., 2012).

There is scant evidence that people spontaneously engage in attention-based refreshing: Self-report studies of strategies during complex-span tasks (e.g., Bailey et al., 2008, 2009; Bailey, Dunlosky, \& Kane, 2011; Dunlosky \& Kane, 2007; Kaakinen \& Hyönä, 2007; St Clair-Thompson, Stevens, Hunt, \& Bolder, 2010) have not revealed "attending to the memoranda" as a strategy people report. Vergauwe and Cowan (2014) have demonstrated that speeded responses in the retention interval of a short-term memory task increase linearly with the memory set size. This result is consistent with the notion that people delay responding by as much time as they need to refresh the memory set once, but it is open to other interpretations, such as a general adjustment of the speed-accuracy settings to the perceived difficulty of the task. In support of that particular alternative interpretation, McCabe (2010) found that response times on the distractor task of a complex-span test increased with the anticipated memory set size already after encoding of the first item.

Direct evidence for a beneficial role of refreshing ${ }_{a t t}$ on at least some form of memory comes from studies by Raye and colleagues (Raye, Johnson, Mitchell, Reeder, \& Greene, 2002; Raye et al., 2007), who asked participants to refresh (i.e., "think about") an occasional word immediately after reading it. On a surprise final recognition test, words that were refreshed were better recognized than words merely read. These studies demonstrate that refreshing improves long-term memory, in line with other research demonstrating that long-term retention depends strongly on whether and how long people attended to the memoranda during encoding (e.g., F. Craik, Govoni, Naveh-Benjamin, \& Anderson, 1996; Phaf \& Wolters, 1993). The only evidence so far that refreshing att 
improves working memory comes from a study on the reproduction of colors from a visual array (Souza et al., 2015), and it is as yet open whether these findings generalize to verbal stimuli, or to serial-recall tests of working memory.

Another open question is whether refreshing att $_{\text {can }}$ proceed as rapidly as was necessary in our simulations in order for refreshing att $_{\text {to }}$ to beneficial for memory performance. Recent empirical work that has adduced support for a rapid pace of refreshing $_{a t t}$ is tied to interpretation within a particular theory (Vergauwe \& Cowan, 2014) and thus does not provide independent, direct evidence. To date, the best available direct evidence for attentional switching has estimated the time for switching from one memory item to another to be around $300 \mathrm{~ms}$ (Garavan, 1998; Oberauer, 2003). Those times are no different from the speed of rehearsal ${ }_{a r t}$, and they underscore the need for

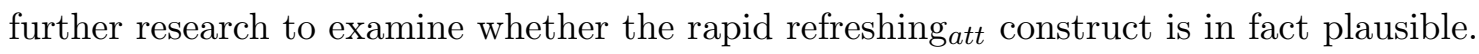

The failure to capture the effects of presentation rate. The beneficial effect of slowed presentation rates reported by Tan and Ward (2008) failed to be captured by both models: The decay model consistently predicted that performance would decrease with slower presentation, whereas SOB-CS predicted a null effect of presentation rate. There is a plethora of evidence that slower presentations give rise to better memory performance for visually presented lists (Dornbush, 1968a, 1968b, 1969; Fell \& Laughery, 1969; Laughery \& Fell, 1969; Mackworth, 1962, 1964; Murray, 1965, 1966; Murray \& Roberts, 1968; Norman, 1966; Sherman \& Turvey, 1969; Woodward, 1970), and the failure to capture that effect thus represents a serious challenge to the models - in particular for SOB-CS, which was not already ruled out by other aspects of the data, such as the shape of the serial position curve. ${ }^{8}$

Upon reflection, it is not surprising that maintenance rehearsal does not show a benefit for slower presentation, because by definition, maintenance rehearsal serves to maintain, not improve, the quality of memory representations. Therefore, we should not 
expect a mechanism of maintenance rehearsal, when added to a model of serial recall, to improve memory with longer durations. The presentation-rate effect observed by Tan and Ward (2008) is therefore likely due to a process that improves the quality of memory above and beyond initial encoding strengths, and enhances its resistance subsequent to encoding. In support, it has long been known that repetition of material in a Brown-Peterson paradigm prior to the onset of the distractor task improves subsequent retention (Hellyer, 1962; Kroll \& Kellicutt, 1972). One well documented process for achieving such an improvement is elaboration of the memoranda, as for example achieved by a levels-of-processing manipulation. There is limited evidence that a levels-of-processing effect can be obtained in a complex-span task, in particular at longer list lengths (Loaiza \& McCabe, 2012; Rose \& Craik, 2012). The precise mechanisms of elaboration are still elusive, but there is some agreement that elaboration makes memory representations more distinctive (e.g., Hunt, 1995).

We therefore constructed a final simulation (Simulation 6) involving the SOB-CS model that included a simple implementation of that idea: We "elaborated" representations of the recall candidates in long-term memory by rendering them more distinctive. In SOB-CS, distinctiveness of memory representations is reflected by the parameter $c$, which governs how sharply the similarity of a recall candidate to the retrieved vector falls off with increasing Euclidean distance between the two vectors. Instead of using the same value of $c$ for all recall candidates, we assigned individual distinctiveness values to each candidate. Initially all candidates receive a relatively low value $(c=0.25)$. By encoding a list item, the corresponding candidate's $c$ value is incremented by a constant amount $(\Delta c=0.04)$. Every time that list item is rehearsed (i.e., every time it is retrieved during rehearsal), its $c$ value is again incremented by the same constant. This continues to happen even if the model stops producing overt 
rehearsal in the fixed-rehearsal schedule, because we assume that when people stop rehearsing aloud, they still continue elaborating.

The result of Simulation 6 with this simple proxy of elaborative rehearsal is shown in Figure 15. The model was simulating the procedures of Tan and Ward (2008), and memory was found to improve with slower presentation rates, in line with the data. We do not present this simulation as a final explanation, but at least the results permit us to suggest that further exploration of the effects of presentation time should focus on the mechanisms of elaboration.

\section{Alternative Conceptions of Rehearsal}

In all our simulations both types of rehearsal involved forward serial retrieval and re-encoding of the retrieved information. The only difference between refreshing att $_{\text {and }}$ rehearsal $_{\text {art }}$ was the speed of retrieval and re-encoding. One might therefore legitimately ask if there are other ways in which rehearsal might be instantiated? Might those other instantiations paint a different picture of rehearsal?

Simultaneous restoration. What if rehearsal involved the failsafe, near-instant, and simultaneous amplification of the contents of memory? If all list items in memory are simultaneously restored to their original strength, would this avoid the introduction of order errors during rehearsal? For rehearsal ${ }_{\text {art }}$, this alternative conception is ruled out fairly convincingly by the fact that overt or covert articulation is necessarily sequential in nature and proceeds at a relatively slow pace - it is logically impossible that a slow sequential process could simultaneously restore all list items.

At first glance, simultaneous restoration thus appears more compatible with

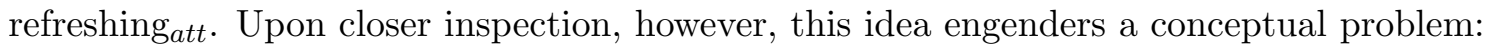
If refreshing were failsafe and simultaneous, then it would be unclear why it would not also be engaged during retrieval from memory - thus yielding virtually perfect recall even 
after prolonged retention intervals, and even when rehearsal ${ }_{a r t}$ is blocked during recall. In a nutshell, if restoration is assumed to be failsafe, then it must be assumed to be independent of the current quality of the memory representation, and then it follows that it can be applied to restore any memory at any time, so that there would be no limit to memory.

Targeted restoration. A more plausible alternative, and one that is closely related to the mechanisms we explored, also involves sequential retrieval and re-encoding of the retrieved information, but the retrieval would target only those items that are most in need of restoration. This alternative is attractive at first glance because it would seem more efficient - the system would not bother to re-encode traces that are uncompromised.

However, this view presumes knowledge of the state of a trace prior to its retrieval: If the state of the trace is a trigger for rehearsal, how can that state be known without a retrieval attempt? And if retrieval is necessary to assess the state of the trace, then this scheme effectively converges with the regime implemented in our simulations.

It follows that a scheme that sequentially retrieves and refreshes all items irrespective of their strength is not entirely arbitrary but conceptually well supported.

Competing models of rehearsal. We are aware of three existing models that have computationally instantiated rehearsal in short-term and working-memory tasks. Burgess and Hitch (1999) implemented a cumulative-forward rehearsal regime in their model. The Burgess and Hitch model is based on positional markers, similar to SOB-CS, but being an instantiation of the "phonological loop", it models forgetting by decay rather than interference. Consonant with our findings, Burgess and Hitch (1999) showed that additional opportunities for rehearsal are associated with an increasing deterioration of performance (their Figure 17). They nonetheless argued for the efficacy of rehearsal based on the fact that the rate of deterioration was decreasing across rehearsals; however, a 
decreasing rate of deterioration is insufficient in light of data that show persistence of information across unfilled (or indeed filled) intervals of considerable duration (Lewandowsky et al., 2004; Vallar \& Baddeley, 1982).

Two models within the ACT-R architecture have also instantiated rehearsal. Anderson et al. (1998) presented a model of list memory that foregrounded decay and rehearsal. Two aspects of their model are particularly relevant here: First, Anderson et al. assumed that the system always commences rehearsal with the first list item and then proceeds through the list from there. This cumulative regime is interleaved with rehearsal of the present item, and once rehearsal has commenced with the first item, the two regimes - continuation of cumulative vs. rehearsal of the current item - are in equal competition with each other at each step. This instantiation of rehearsal if at odds with the data reported by Tan and Ward (2008) for short presentation times: People are known to rehearse only the current item when presentation is rapid, whereas the model of Anderson et al. (1998) begins rehearsal with the first item in all cases. Its results are thus not directly comparable to the present simulations which were guided by the behaviorally observed rehearsal patterns. In particular, the fact that Anderson et al. (1998) showed plausible serial position curves with rehearsal, even at short presentation times of $1 \mathrm{~s} /$ item, likely arose from their (now known to be unrealistic) rehearsal pattern. The performance of their model was further aided by the way in which order was encoded in their model: Each list item was represented by an integrated chunk involving the item coupled to its list position (there were also other hierarchical chunks that we ignore here for simplicity). In consequence, although the model might fail to retrieve an item-to-position chunk during a given rehearsal or recall step, or might retrieve an incorrect item-to-position chunk, there was no way that rehearsal could mistakenly associate an item in position $j$ to any other position $k \neq j$. 
Daily et al. (2001) presented another model within the ACT-R family that modeled performance in a complex-span task. This model used a similar atomic representational scheme to that used by Anderson et al. (1998); that is, the model encoded item-to-position chunks whose activations declined through decay but were boosted by rehearsal. The model thus inherits the property that rehearsal cannot actually re-order list items, because even if the wrong item-to-position association is picked during rehearsal, upon its strengthening the item is still associated with its original position.

\section{Implications for Models of Memory}

We begin by considering decay models (e.g., Barrouillet et al., 2004; Oberauer \& Lewandowsky, 2011; Page \& Norris, 1998) which appear to be particularly challenged by our simulation results. Perhaps most critically, when simulated rehearsal patterns follow the distribution observed in the data (Simulation 1; Figure 6), the decay model failed to produce serial position curves that resembled the data (Figure 7). The model became competitive only when rehearsal was replaced by (cumulative) attentional refreshing, in which case it handled several results at least at a coarse level (e.g., cognitive load in complex span in Simulation 3).

Another adverse implication of our results for decay models concerns the role of articulatory suppression. The decline in memory performance that is observed when people engage in overt articulation during a memory trial is conventionally ascribed to the prevention of articulatory rehearsal (e.g., Burgess \& Hitch, 1999). Our simulation results suggest instead that prevention of articulatory rehearsal should be expected to improve memory performance under conditions that allow simultaneous refreshing (see, e.g., Figure 8). It follows that the adverse impact of articulatory suppression is better explained by some other source, such as interference between phonological representations. 
An interference-based approach to AS meshes well with earlier modeling and the results of our simulation using the SOB-CS model: Unlike the decay model, the performance of SOB-CS was not impaired by additional opportunity for maintenance rehearsal (Simulation 5), and when an elaborative rehearsal mechanism was added to the model, it was able to reproduce the observed positive effect of presentation duration (Simulation 6). Given that the same model is known to accommodate several benchmark results from short-term (Lewandowsky \& Farrell, 2008a, 2008b; Oberauer \& Lewandowsky, 2008) and working memory (Oberauer et al., 2012), including results that are often cited in support of decay models, the present simulations buttress the standing of SOB-CS as a viable interference-based approach to modeling of memory.

It must be noted that SOB-CS is not the only theory to explain a variety of memory phenomena on the basis of interference, rather than decay, and without a necessary appeal to rehearsal. Farrell (2012) presented a theory that was based on the notion that people parse the continuous stream of experiences into distinct "episodes", and that items within an episode are tied to a common temporal context. Farrell's model does not require rehearsal to explain a number of memory phenomena - including serial recall and free recall over the short and the long term — although it acknowledges the possibility that elaboration might assist recall, especially after a delay. It is conceivable that in Farrell's

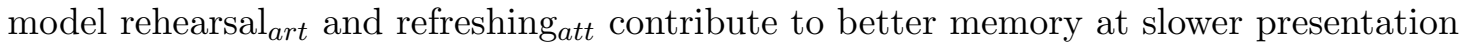
times. ${ }^{9}$ For instance, lists of 6 items might be broken into short episodes of two or three

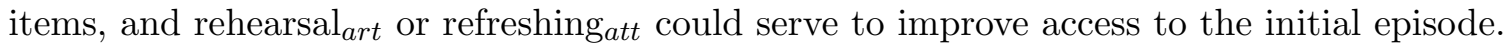

\section{Conclusions}

Our principal conclusion is that a reflexive appeal to rehearsal in order to explain a memory phenomenon is not an explanation - it can only be the beginning of a process of examination that may or may not converge on rehearsal as an underlying explanatory 
process. We have shown that in some circumstances it is inadvisable to appeal to rehearsal: For example, when no further forgetting is observed if an unfilled delay between encoding and recall is extended (Lewandowsky et al., 2004), this maintenance of memory cannot be explained by articulatory rehearsal because extending rehearsal ${ }_{\text {art }}$ would be predicted to make memory worse (Simulation 4). The absence of time-based forgetting in these conditions must therefore be explained without appeal to articulatory rehearsal.

A second conclusion that emerges from our work is that although rehearsal is routinely invoked by decay theorists, our decay-plus-rehearsal model had considerable difficulty reproducing some fairly basic results from the literature, notwithstanding its ability to capture human overt rehearsal patterns quite accurately. We cannot rule out that there are other implementations of rehearsal that work better within a decay framework, but the onus is now on decay-rehearsal theorists to present such an alternative implementation. In the meantime, however, we point out that within the SOB-CS theory, rehearsal art did not cause the same deleterious effects that arose within a decay framework. Seen in a positive light, this result simplifies future theorizing because a single explanatory process - viz. interference - may replace the twin processes of decay and rehearsal, at least in simple span. 
Rehearsal and Short-Term Retention 56

\section{References}

Allen, R., \& Hulme, C. (2006). Speech and language processing mechanisms in verbal serial recall. Journal of Memory and Language, 55, 64-88.

Alloway, T. P., Gathercole, S. E., \& Pickering, S. J. (2006). Verbal and visuospatial short-term and working memory in children: Are they separable? Child Development, 77, 1698-1716.

Anderson, J. R., Bothell, D., Lebiere, C., \& Matessa, M. (1998). An integrated theory of list memory. Journal of Memory and Language, 38, 341-380.

Baddeley, A. D. (1986). Working memory. New York: Oxford University Press.

Baddeley, A. D., \& Hitch, G. (1974). Working memory. In G. Bower (Ed.), The psychology of learning and motivation: Advances in research and theory (vol. 8, pp. 47-89). New York: Academic Press.

Baddeley, A. D., Thomson, N., \& Buchanan, M. (1975). Word length and structure of short-term memory. Journal of Verbal Learning and Verbal Behavior, 14, 575-589.

Bailey, H., Dunlosky, J., \& Hertzog, C. (2009). Does differential strategy use account for age-related deficits in working-memory performance? Psychology and Aging, 24, 82-92. doi: $10.1037 / \mathrm{a} 0014078$

Bailey, H., Dunlosky, J., \& Kane, M. J. (2008). Why does working memory span predict complex cognition? testing the strategy affordance hypothesis. Memory $\mathbb{6}$ Cognition, 36, 1383-1390. doi: 10.3758/MC.36.8.1383

Bailey, H., Dunlosky, J., \& Kane, M. J. (2011). Contribution of strategy use to performance on complex and simple span tasks. Memory $\&$ Cognition, 39, 447-461. doi: 10.3758/s13421-010-0034-3

Barrouillet, P., Bernardin, S., \& Camos, V. (2004). Time constraints and resource sharing in adults' working memory spans. Journal of Experimental Psychology: General, 133, 83-100. 
Barrouillet, P., Bernardin, S., Portrat, S., Vergauwe, E., \& Camos, V. (2007). Time and cognitive load in working memory. Journal of Experimental Psychology: Learning, Memory \& Cognition, 33, 570-585.

Bhatarah, P., Ward, G., Smith, J., \& Hayes, L. (2009). Examining the relationship between free recall and immediate serial recall: Similar patterns of rehearsal and similar effects of word length, presentation rate, and articulatory suppression. Memory \& Cognition, 37, 689-713.

Botvinick, M. M., Braver, T. S., Barch, D. M., Carter, C. S., \& Cohen, J. D. (2001). Conflict monitoring and cognitive control. Psychological Review, 108, 624-652.

Brown, G. D. A., Preece, T., \& Hulme, C. (2000). Oscillator-based memory for serial order. Psychological Review, 107, 127-181.

Brown, J. (1958). Some tests of the decay theory of immediate memory. Quarterly Journal of Experimental Psychology, 10, 12-21.

Burgess, N., \& Hitch, G. J. (1999). Memory for serial order: A network model of the phonological loop and its timing. Psychological Review, 106, 551-581.

Burgess, N., \& Hitch, G. J. (2006). A revised model of short-term memory and long-term learning of verbal sequences. Journal of Memory and Language, 55, 627-652.

Camos, V., Lagner, P., \& Barrouillet, P. (2009). Two maintenance mechanisms of verbal information in working memory. Journal of Memory and Language, 61, 457-469.

Conway, A. R. A., Kane, M. J., Bunting, M. F., Hambrick, D. Z., Wilhelm, O., \& Engle, R. W. (2005). Working memory span tasks: A methodological review and user's guide. Psychonomic Bulletin \& Review, 12, 769-786.

Craik, F., Govoni, R., Naveh-Benjamin, M., \& Anderson, N. (1996). The effects of divided attention on encoding and retrieval processes in human memory. Journal of Experimental Psychology: General, 125, 159-80.

Craik, F. I. M., \& Lockhart, R. S. (1972). Levels of processing: A framework for memory 
research. Journal of Verbal Learning \& Verbal Behavior, 11, 671-684.

Craik, F. I. M., \& Tulving, E. (1975). Depth of processing and the retention of words in episodic memory. Journal of Experimental Psychology: General, 104, 268-294.

Daily, L., Lovett, M., \& Reder, L. (2001). Modeling individual differences in working memory performance: A source activation account. Cognitive Science, 25, 315-353.

Daneman, M., \& Carpenter, P. (1980). Individual differences in working memory and reading. Journal of Verbal Learning \& Verbal Behavior, 19, 450-466.

Della Sala, S., Cowan, N., Beschin, N., \& Perini, M. (2005). Just lying there, remembering: Improving recall of prose in amnesic patients with mild cognitive impairment by minimising interference. Memory, 13, 435-440. doi: $10.1080 / 09658210344000387$

Dornbush, R. L. (1968a). Input variables in bisensory memory. Perception $\mathcal{E}^{3}$ Psychophysics, 4, 41-44. doi: 10.3758/BF03210445

Dornbush, R. L. (1968b). Shadowing in bisensory memory. Quarterly Journal of Experimental Psychology, 20, 225-231. doi: 10.1080/14640746808400156

Dornbush, R. L. (1969). Stimulus information and stimulus interference in bisensory short-term memory. Perception \& Psychophysics, 5, 303-304.

Dosher, B. A., \& Ma, J. J. (1998). Output loss or rehearsal loop? output-time versus pronunciation-time limits in immediate recall for forgetting-matched materials. Journal of Experimental Psychology: Learning, Memory, and Cognition, 24, 316-335.

Dunlosky, J., \& Kane, M. J. (2007). The contributions of strategy use to working memory span: A comparison of strategy-assessment methods. Quarterly Journal of Experimental Psychology, 60, 1227-1245.

Ecker, U. K. H., Lewandowsky, S., \& Oberauer, K. (2013). Removal of information from working memory: A specific updating process. Journal of Memory and Language. 
Ecker, U. K. H., Oberauer, K., \& Lewandowsky, S. (2014). Working memory updating involves item-specific removal. Journal of Memory and Language, 74, 1-15.

Farrell, S. (2012). Temporal clustering and sequencing in short-term memory and episodic memory. Psychological Review, 119, 223-271.

Farrell, S., \& Lewandowsky, S. (2002). An endogenous distributed model of ordering in serial recall. Psychonomic Bulletin \& Review, 9, 59-79.

Farrell, S., \& Lewandowsky, S. (2003). Dissimilar items benefit from phonological similarity in serial recall. Journal of Experimental Psychology: Learning, Memory, and Cognition, 29, 838-49.

Farrell, S., \& Lewandowsky, S. (2004). Modelling transposition latencies: Constraints for theories of serial order memory. Journal of Memory and Language, 51, 115-135.

Fawcett, J. M., \& Taylor, T. L. (2008). Forgetting is effortful: Evidence from reaction time probes in an item-method directed forgetting task. Memory $\&$ Cognition, 36 , $1168-1181$.

Fell, J. C., \& Laughery, K. R. (1969). Short-term memory: Mode of presentation for alphanumeric information. Human Factors, 11, 401-405.

Garavan, H. (1998). Serial attention within working memory. Memory 83 Cognition, 26, 263-276.

Gathercole, S. E., Pickering, S. J., Ambridge, B., \& Wearing, H. (2004). The structure of working memory from 4 to 15 years of age. Developmental Psychology, 40, 177-190.

Gerver, D. (1969). Effects of grammaticalness, presentation rate, and message length of auditory short-term memory. Quarterly Journal of Experimental Psychology, 21, 203-208. doi: 10.1080/14640746908400214

Greenwald, A. G. (1970). A choice reaction time test of ideomotor theory. Jouirnal of Experimental Psychology, 86, 20-25.

Grenfell-Essam, R., \& Ward, G. (2012). Examining the relationship between free recall 
and immediate serial recall: The role of list length, strategy use, and test expectancy. Journal of Memory and Language, 67, 106-148. doi: 10.1016/j.jml.2012.04.004

Grenfell-Essam, R., Ward, G., \& Tan, L. (2012). The role of rehearsal on the output order of immediate free recall of short and long lists. Journal of Experimental Psychology: Learning, Memory, and Cognition, 1-31. doi: 10.1037/a0028974

Gupta, P., \& MacWhinney, B. (1995). Is the articulatory loop articulatory or auditory? reexamining the effects of concurrent articulation on immediate serial recall. Journal of Memory and Language, 34, 63-88.

Hellyer, S. (1962). Supplementary report: Frequency of stimulus presentation and short-term decrement in recall. Journal of Experimental Psychology, 64(6), 650.

Henson, R. N. A. (1998). Short-term memory for serial order: The Start-End model. Cognitive Psychology, 36, 73-137.

Henson, R. N. A., Norris, D. G., Page, M. P. A., \& Baddeley, A. D. (1996). Unchained memory: Error patterns rule out chaining models of immediate serial recall. Quarterly Journal of Experimental Psychology, 49A, 80-115.

Hommel, B. (2004). Coloring an action: Intending to produce color events eliminates the stroop effect. Psychological Research, 68, 74-90. doi: 10.1007/s00426-003-0146-5

Hudjetz, A., \& Oberauer, K. (2007). The effects of processing time and processing rate on forgetting in working memory: Testing four models of the complex span paradigm. Memory \& Cognition, 35, 1675-1684.

Humphreys, M. S., Maguire, A. M., McFarlane, K. A., Burt, J. S., Bolland, S. W., Murray, K. L., \& Dunn, R. (2010). Using maintenance rehearsal to explore recognition memory. Journal of Experimental Psychology: Learning, Memory, and Cognition, 36, 147-159.

Hunt, R. (1995). The subtlety of distinctiveness: What von restorff really did. Psychonomic Bulletin \& Review, 2, 105-112. 
Hurlstone, M. J., Hitch, G. J., \& Baddeley, A. D. (2013). Memory for serial order across domains: An overview of the literature and directions for future research. Psychological Bulletin.

Jalbert, A., Neath, I., Bireta, T. J., \& Surprenant, A. M. (2011). When does length cause the word length effect? Journal of Experimental Psychology: Learning, Memory, \& Cognition, 37, 338-353.

Jarrold, C., \& Hall, D. (2012). The development of rehearsal in verbal short-term memory. Manuscript submitted for publication.

Jarrold, C., \& Tam, H. (2011). Rehearsal and the development of working memory. In P. Barrouillet \& V. Gaillard (Eds.), Cognitive development and working memory: A dialogue between neo-Piagetian and cognitive approaches (pp. 177-199). Hove, UK: Psychology Press.

Jolicoeur, P., \& Dell'Acqua, R. (1998). The demonstration of short-term consolidation. Cognitive Psychology, 36, 138-202.

Kaakinen, J. K., \& Hyönä, J. (2007). Strategy use in the reading span test: An analysis of eye movements and reported encoding strategies. Memory, 15, 634-646.

Kieras, D. E., Meyer, D. E., Mueller, S., \& Seymour, Y. (1999). Models of working memory. In A. Miyake \& P. Shah (Eds.), (pp. 183-223). Cambridge, England: Cambridge University Press.

Kroll, N. E., \& Kellicutt, M. (1972). Short-term recall as a function of covert rehearsal and of intervening task. Journal of Verbal Learning and Verbal Behavior, 11, 196 204. doi: $10.1016 / \mathrm{S} 0022-5371(72) 80077-\mathrm{X}$

Laming, D. (2008). An improved algorithm for predicting free recalls. Cognitive Psychology, 57, 179-219.

Laming, D. (2010). Serial position curves in free recall. Psychological Review, 117, $93-133$. 
Laughery, K. R., \& Fell, J. C. (1969). Subject preferences and nature of information stored in short-term memory. Journal of Experimental Psychology, 82, 193-197. doi: $10.1037 / \mathrm{h} 0028149$

Lewandowsky, S., Duncan, M., \& Brown, G. D. A. (2004). Time does not cause forgetting in short-term serial recall. Psychonomic Bulletin \& Review, 11, 771-790.

Lewandowsky, S., \& Farrell, S. (2008a). Phonological similarity in serial recall: Constraints on theories of memory. Journal of Memory and Language, 58, 429-448. Lewandowsky, S., \& Farrell, S. (2008b). Short-term memory: New data and a model. In B. H. Ross (Ed.), The psychology of learning and motivation (Vol. 49, pp. 1-48). London, UK: Elsevier.

Lewandowsky, S., Geiger, S. M., Morrell, D. B., \& Oberauer, K. (2010). Turning simple span into complex span: Time for decay or interference from distractors? Journal of Experimental Psychology: Learning, Memory, and Cognition, 36, 958-978.

Lewandowsky, S., Geiger, S. M., \& Oberauer, K. (2008). Interference-based forgetting in verbal short-term memory. Journal of Memory and Language, 59, 200-222.

Lewandowsky, S., \& Li, S.-C. (1994). Memory for serial order revisited. Psychological Review, 101, 539-543.

Lewandowsky, S., \& Murdock, B. B. (1989). Memory for serial order. Psychological Review, 96, 25-57.

Lewandowsky, S., \& Oberauer, K. (2008). The word length effect provides no evidence for decay in short-term memory. Psychonomic Bulletin $\&$ Review, 15, 875-888.

Lewandowsky, S., \& Oberauer, K. (2009). No evidence for temporal decay in working memory. Journal of Experimental Psychology: Learning, Memory, and Cognition, $35,1545-1551$

Lewandowsky, S., Oberauer, K., \& Brown, G. D. A. (2009). No temporal decay in verbal short-term memory. Trends in Cognitive Sciences, 13, 120-126. 
Lewandowsky, S., Oberauer, K., Yang, L.-X., \& Ecker, U. K. H. (2010). A working memory test battery for MATLAB. Behavior Research Methods, 42, 571-585.

Loaiza, V. M., \& McCabe, D. P. (2012). Temporal-contextual processing in working memory: Evidence from delayed cued recall and delayed free recall tests. Memory $\&$ Cognition, 40, 191-203.

Longoni, A., Richardson, J., \& Aiello, A. (1993). Articulatory rehearsal and phonological storage in working memory. Memory $\&$ Cognition, 21, 11-22.

Mackworth, J. F. (1962). The effect of display time upon the recall of digits. Canadian Journal of Psychology, 16, 48-54. doi: 10.1037/h0083241

Mackworth, J. F. (1964). Auditory short-term-memory. Canadian Journal of Psychology, $18,292-303$.

McCabe, D. P. (2010). The influence of complex working memory span task administration methods on prediction of higher level cognition and metacognitive control of response times. Memory \& Cognition, 38, 868-882. doi: 10.3758/MC.38.7.868

McFarlane, K. A., \& Humphreys, M. S. (2012). Maintenance rehearsal: The key to the role attention. Jouirnal of Experimental Psychology: Learning, Memory, and Cognition. doi: 10.1037/a0026783

Milner, B. (1970). Memory and the medial temporal regions of the brain. In K. H. Pribram \& B. D. E. (Eds.), Biology of memory. New York: Academic Press. Murray, D. J. (1965). Vocalization-at-presentation and immediate recall, with varying presentation-rates. Quarterly Journal of Experimental Psychology, 17, 47-56. doi: $10.1080 / 17470216508416407$

Murray, D. J. (1966). Vocalization-at-presentation and immediate recall with varying recall methods. Quarterly Journal of Experimental Psychology, 17, 47-56. doi: $10.1080 / 14640746608400002$ 
Murray, D. J. (1967). The role of speech responses in short-term memory. Canadian Journal of Psychology, 21, 263-276.

Murray, D. J., \& Roberts, B. (1968). Visual and auditory presentation, presentation rate and short-term memory in children. British Journal of Psychology, 59, 119-125.

Norman, D. A. (1966). Acquisition and retention in short-term memory. Journal of Experimental Psychology, 72, 369-381. doi: 10.1037/h0023647

Oberauer, K. (2002). Access to information in working memory: Exploring the focus of attention. Journal of Experimental Psychology: Learning, Memory, and Cognition, $28,411-421$.

Oberauer, K. (2003). Selective attention to elements in working memory. Experimental Psychology, 50, 257-269.

Oberauer, K., \& Lewandowsky, S. (2008). Forgetting in immediate serial recall: Decay, temporal distinctiveness, or interference? Psychological Review, 115, 544-576.

Oberauer, K., \& Lewandowsky, S. (2011). Modeling working memory: a computational implementation of the time-based resource-sharing theory. Psychonomic Bulletin 86 Review, 18, 10-45.

Oberauer, K., \& Lewandowsky, S. (2014). Further evidence against decay in working memory. Journal of Memory and Language, 73, 15-30.

Oberauer, K., Lewandowsky, S., Farrell, S., Jarrold, C., \& Greaves, M. (2012). Modeling working memory: An interference model of complex span. Psychonomic Bulletin $\mathbb{E}$ Review, 19, 779-819.

Olivers, C. N. L., Meijer, F., \& Theeuwes, J. (2006). Feature-based memory-driven attentional capture: Visual working memory content affects visual attention. Journal of Experimental Psychology: Human Perception \& Performance, 32, 1243-1265.

Page, M. P. A., \& Norris, D. (1998). The primacy model: A new model of immediate serial recall. Psychological Review, 105, 761-781. 
Peterson, L. R., \& Peterson, M. J. (1959). Short-term retention of individual verbal items. Journal of Experimental Psychology, 58, 193-198.

Phaf, R. H., \& Wolters, G. (1993). Attention shifts in maintenance rehearsal. The Americal Journal of Psychology, 106, 353-382.

Posner, M. I. (1964). Rate of presentation and order of recall in immediate memory. British Journal of Psychology, 55, 303-306.

Raaijmakers, J. G. W., \& Shiffrin, R. M. (1981). Search of associative memory. Psychological Review, 88, 93-134.

Raye, C., Johnson, M., Mitchell, K., Greene, E., \& Johnson, M. (2007). Refreshing: A minimal executive function. Cortex, 43, 135-145.

Raye, C., Johnson, M., Mitchell, K., Reeder, J., \& Greene, E. (2002). Neuroimaging a single thought: Dorsolateral pfc activity associated with refreshing just-activated information. NeuroImage, 15, 447-453.

Ricker, T. J., \& Cowan, N. (2010). Loss of visual working memory within seconds: The combined use of refreshable and non-refreshable features. Journal of Experimental Psychology-learning Memory and Cognition, 36, 1355-1368. doi: 10.1037/a0020356

Ricker, T. J., Cowan, N., \& Morey, C. (2010). Visual working memory is disrupted by covert verbal retrieval. Psychonomic Bulletin \& Review, 17, 516-521. doi: 10.3758/PBR.17.4.516

Rose, N. S., \& Craik, F. I. M. (2012). A processing approach to the working memory/long-term memory distinction: Evidence from the levels-of-processing span task. Journal of Experimental Psychology: Learning Memory and Cognition, 38, 1019-1029. doi: 10.1037/a0026976

Rose, N. S., Myerson, J., Roediger, I., Henry L., \& Hale, S. (2010). Similarities and differences between working memory and long-term memory: Evidence from the levels-of-processing span task. Journal of Experimental Psychology: Learning 
Memory and Cognition, 36, 471-483. doi: 10.1037/a0018405

Rundus, D. (1971). Analysis of rehearsal processes in free recall. Journal of Experimental Psychology, 89, 63-77.

Sherman, M. F., \& Turvey, M. T. (1969). Modality differences in short-term serial memory as a function of presentation rate. Journal of Experimental Psychology, 80, 335-338. doi: 10.1037/h0027276

Soto, D., Heinke, D., Humphreys, G. W., \& Blanco, M. (2005). Early, involuntary top-down guidance of attention from working memory. Journal of Experimental Psychology: Human Perception and Performance, 31, 248-261. doi: 10.1037/0096-1523.31.2.248

Souza, A. S., Rerko, L., \& Oberauer, K. (2015). Refreshing memory traces: thinking of an item improves retrieval from visual working memory. Annals of the New York Academy of Sciences.

St Clair-Thompson, H., Stevens, R., Hunt, A., \& Bolder, E. (2010). Improving children's working memory and classroom performance. Educational Psychology, 30, 203-219. doi: $10.1080 / 01443410903509259$

Tan, L., \& Ward, G. (2000). A recency-based account of the primacy effect in free recall. Journal of Experimental Psychology: Learning, Memory, and Cognition, 26, $1589-1625$.

Tan, L., \& Ward, G. (2008). Rehearsal in immediate serial recall. Psychonomic Bulletin E Review, 15, 535-542.

Turley-Ames, K. J., \& Whitfield, M. M. (2003). Strategy training and working memory task performance. Journal of Memory and Language, 49, 446-468.

Vallar, G., \& Baddeley, A. D. (1982). Short-term forgeting and the articulatory loop. Quarterly Journal of Experimental Psychology, 34A, 53-60.

Vergauwe, E., Camos, V., \& Barrouillet, P. (2014). The impact of storage on processing: 
How is information maintained in working memory? Journal of Experimental Psychology: Learning, Memory, and Cognition, 40, 1072-1095.

Vergauwe, E., \& Cowan, N. (2014). A common short-term memory retrieval rate may describe many cognitive procedures. Frontiers in Human Neuroscience, 8. doi: 10.3389/fnhum.2014.00126

Ward, G., Tan, L., \& Grenfell-Essam, R. (2010). Examining the relationship between free recall and immediate serial recall: The effects of list length and output order. Journal of Experimental Psychology: Learning, Memory, and Cognition, 36, $1207-1241$.

Watkins, M. J., \& Peynircioglu, Z. F. (1982). A perspective on rehearsal. In G. H. Bower (Ed.), Psychology of learning and motivation (Vol. 16, pp. 153-190). New York: Academic Press.

Woodward, A. E. (1970). Continuity between serial memory and serial learning. Journal of Experimental Psychology, 85, 90-94. doi: 10.1037/h0029541 


\section{Appendix}

\section{Differentiating item errors in SOB-CS}

Errors in serial recall can be broadly subdivided into order errors (i.e., recalling a list item in the wrong position) and item errors (all other errors). When participants are allowed to omit responses, item errors can be further subdivided into extra-list intrusions (i.e., recall of an item that was not on the list) and omissions. To date, omissions have not been modeled separately in SOB-CS because they are often disallowed in complex-span experiments. In the study by Tan and Ward, by contrast, the large majority of item errors were omissions (see top panel of Table A1).

Moreover, during rehearsal people are free to "omit" a list item; that is, they can refrain from producing (subvocally or overtly) an item that they cannot retrieve with sufficient confidence. Omitting an item in rehearsal implies that that item is not re-encoded, whereas an error of commission (i.e., an order error or an extralist intrusion) implies that the wrong item is re-encoded, potentially compromising the memory representation. We therefore implemented omissions and extralist intrusions as follows. Retrieval in SOB-CS proceeds in two steps. In the first step, an approximation of the originally-encoded item is re-created using the current position marker as a retrieval cue. In the second step, this retrieved approximation is compared to the representations of all recall candidates in semantic memory (e.g., when the memory list consists of consonants, all consonants are the recall candidates). Because we are modeling lists of words, we arbitrarily defined a set of 81 words as recall candidates. Each recall candidate receives a similarity value, and candidates with a high similarity to the retrieved vector are more likely to be chosen for recall. We assumed that an omission would occur if the similarity comparison failed to yield a single response but instead produced several conflicting candidates. Retrieval conflict was calculated as the Hopfield energy of all similarity values 
(Botvinick, Braver, Barch, Carter, \& Cohen, 2001). It is low when only one recall candidate has a high similarity to the retrieved vector, and it increases with the number of candidates that have high similarities to the retrieved vector. Confidence is computed as the inverse of retrieval conflict, and an omission occurred when confidence failed to exceed a threshold (a free parameter set to 0.04).

To model the approximate prevalence of extralist intrusions, we weighted intralist and extralist recall candidates differentially: Whereas the list members among the recall candidates received the default weight of 1 , the remaining recall candidates in the vocabulary that were not elements of the current list received a reduced weight (a free parameter set to 0.15$)$. These weights are multiplied with the similarities of the recall candidates to the retrieved vector. Those two modifications enabled the model to produce the correct proportions of the three error categories (see bottom panel of Table A1). 
Rehearsal and Short-Term Retention 70

\begin{abstract}
Author Note
Preparation of this paper was facilitated by a Discovery Grant from the Australian Research Council to both authors and an Australian Professorial Fellowship and a Discovery Outstanding Researcher Award to the first author. The first author was also supported by a Royal Society Wolfson Research Merit Award. Correspondence to the first author at the School of Experimental Psychology, University of Bristol, 12A Priory Road, Bristol BS8 1TU, United Kingdom (stephan.lewandowsky@bristol.ac.uk). Personal web page: http://www.cogsciwa.com.
\end{abstract}




\section{Footnotes}

1 One objection to this argument might point to the possibility that articulatory representations are not required for serial recall when the list is not spoken but recalled by other means, such as typing on the keyboard or through a reconstruction task. This objection can be countered by noting that even when visually-presented lists are encoded silently, a phonological similarity effect can be detected with keyboard recall or reconstruction (Farrell \& Lewandowsky, 2003; Lewandowsky \& Farrell, 2008a). This can only be explained by the idea that participants silently articulate the memoranda during encoding, thereby creating articulatory representations that underlie memory irrespective of output modality.

2 An anonymous reviewer raised the interesting possibility that the two modes of rehearsal may be super-additive. This idea assumes that there is some memory for, say, phonological features and some memory for semantic features, with one rehearsal process dedicated to each. The reconstruction of a target from those two cues might be successful even when each cue on its own is insufficient. On this scenario, rehearsal ${ }_{a r t}$ and refreshing ${ }_{a t t}$ would be super-additive. However, like the additive scenario, super-additivity would imply that blocking only one of the two rehearsal mechanisms is sufficient to impair memory. The arguments against additivity thus transfer nearly seamlessly to super-additivity.

3 We thank Pierre Barrouillet for drawing our attention to the analogy between this issue and Newton's work.

${ }^{4}$ In a further simulation, we also implemented the regime that governed rehearsal in Simulation 1, which reproduced the rehearsal patterns identified by Tan and Ward (2008). With this alternative regime the model's performance was again found to be extremely poor whenever rehearsal $l_{a r t}$ was present, and we therefore do not report the results of this simulation. 
5 Following a suggestion by Valerie Camos, we also implemented the dual-rehearsal mechanism in a second way. In this simulation, each item was represented twice in two separate layers that were copies of each other. One representation was regarded as phonological and was affected by rehearsal ${ }_{a r t}$, the other was non-phonological and affected

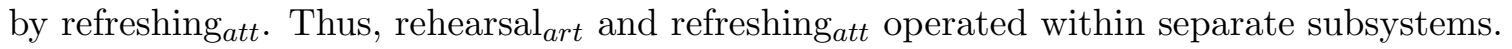
Only at overt recall was information from both subsystems integrated: In the competitive-cueing stage, the activation of the phonological and the non-phonological representation that each item received through positional cueing was added. This simulation yielded results that were virtually indistinguishable from those presented in Figure 10.

${ }^{6}$ In the present simulations, this problem is exacerbated by the fact that response suppression is absent during rehearsal. However, in another simulation (not reported in detail here) in which every just-rehearsed item was temporarily removed from the set of retrieval candidates during the next retrieval step, thereby instantiating a form of response suppression that did not undo the associative boost from rehearsal, the problem was found to persist, albeit in slightly attenuated magnitude.

7 We thank Geoff Ward for sharing with us the raw data from the Tan and Ward (2008) experiment.

8 The pattern is far less clear for auditory presentation, for which any possible outcome has been observed: In some cases slower presentation has improved performance (e.g., Fell \& Laughery, 1969; Gerver, 1969; Laughery \& Fell, 1969; Norman, 1966), in some cases a null effect was observed (e.g., Murray \& Roberts, 1968), and in other instances performance declined as presentation was slowed (e.g., Dornbush, 1968a, 1968b, 1969; Mackworth, 1964; Posner, 1964). Given this as-yet unexplained diversity of outcomes, and given that no studies have overtly observed rehearsal with auditory presentation, we do not consider these results here. 
Rehearsal and Short-Term Retention 73

${ }^{9}$ We thank an anonymous reviewer for pointing out this possibility to us. 
Rehearsal and Short-Term Retention 74

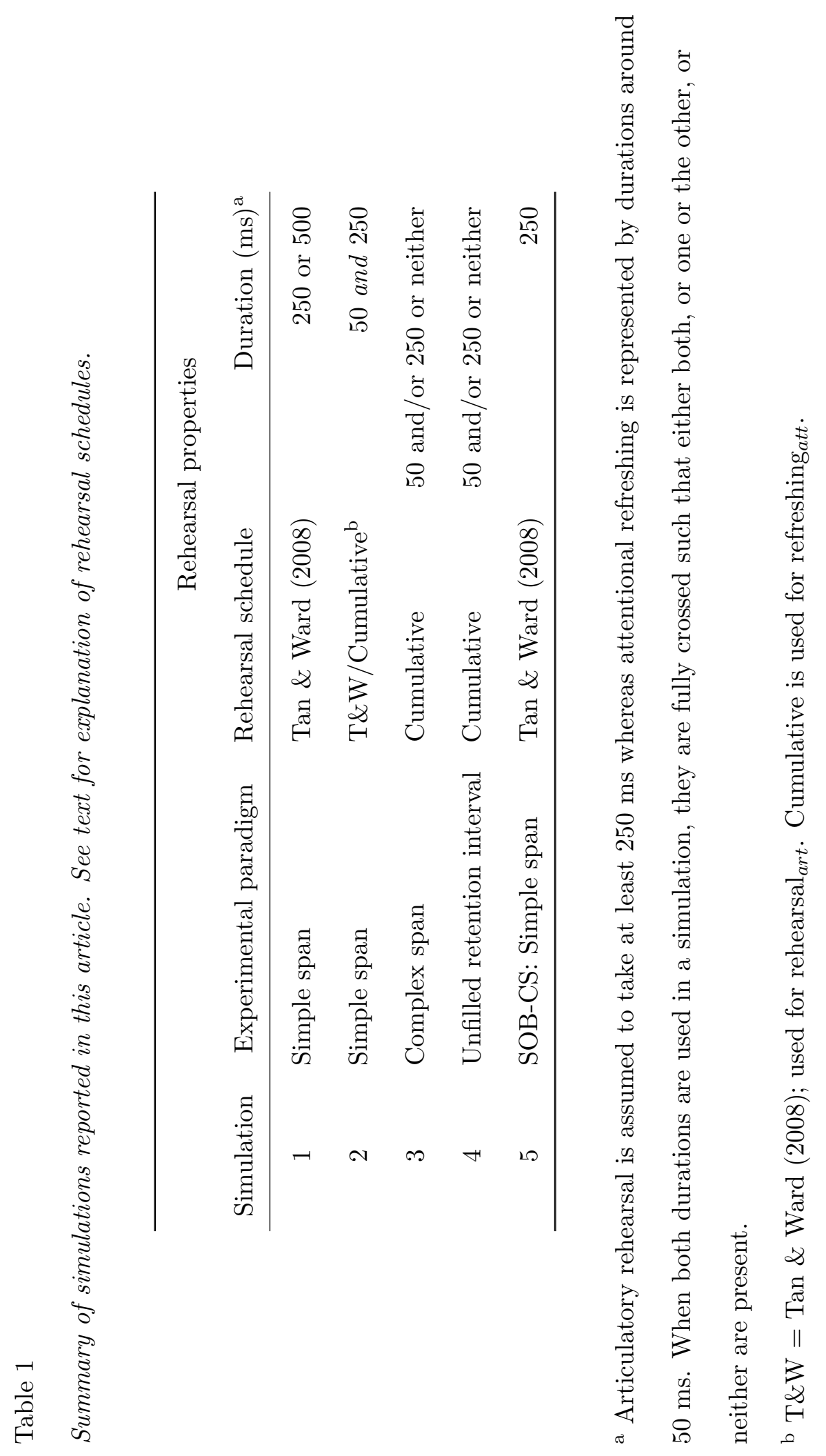


Table 2

Parameter values that were fixed for all simulations. Variable parameter values are provided in the text.

\begin{tabular}{lrr}
\hline \multicolumn{1}{c}{ Name } & Symbol & Value \\
\hline Position overlap & & 0.3 \\
Encoding and processing rate & $R$ & 6 \\
Rate standard deviation & $s$ & 1 \\
Retrieval threshold & & 0.05 \\
Noise & $p$ & 0.02 \\
Primacy gradient & 0.9 \\
Mean encoding duration & $0.5 \mathrm{~s}$ \\
Mean distractor operation duration & $0.5 \mathrm{~s}$ \\
Mean recall time & $0.5 \mathrm{~s}$ \\
\hline
\end{tabular}


Table 3

Proportion of virtual rehearsal errors observed in Simulation 4.

\begin{tabular}{lcrrr}
\hline & & \multicolumn{3}{c}{ Response type $^{\mathrm{a}}$} \\
Condition & Retention interval & Correct & Transpositions & Repetition errors \\
\hline Rehearsal $_{\text {art }}$ & Short & .49 & .02 & .50 \\
Rehearsal $_{\text {art }}$ & Long & .43 & .01 & .56 \\
Rehearsal $_{\text {art }}+$ refreshing $_{a t t}$ & Short & .73 & .02 & .26 \\
Rehearsal $_{\text {art }}+$ refreshing $_{a t t}$ & Long & .71 & .02 & .28 \\
\hline
\end{tabular}

aTable entries are proportions of response type observed across all rehearsal events in each condition. Extralist intrusions did not occur in any condition and are therefore omitted. 
Rehearsal and Short-Term Retention 77

Table A1

Proportion of correct responses and error categories in the experiment of Tan 8 Ward (2008)

(top panel) and in the simulation of $S O B-C S$ (bottom panel)

\begin{tabular}{lccc}
\hline & \multicolumn{3}{c}{ Presentation speed } \\
Response type & Fast & Medium & Slow \\
\hline Tan \& Ward (2008) & & & \\
Correct & .39 & .47 & .56 \\
Transposition & .28 & .23 & .21 \\
Extralist Intrusion & .04 & .04 & .03 \\
Omission & .29 & .26 & .20 \\
& & & \\
SOB-CS & & & \\
Correct & .43 & .52 & .54 \\
Transposition & .24 & .20 & .19 \\
Extralist Intrusion & .02 & .03 & .03 \\
Omission & .31 & .25 & .23 \\
\hline
\end{tabular}




\section{Figure Captions}

Figure 1. Panel A: Observed effects on memory of varying the retention interval, filled with constant AS ("the ...the ...the"). Data are from Humphreys et al. (2010); Lewandowsky et al. (2004, 2008); Lewandowsky, Geiger, et al. (2010); Longoni et al. (1993); McFarlane \& Humphreys (2012); Phaf \& Wolters (1993); Vallar \& Baddeley (1982). Extending the duration of a distractor interval does not lead to increasing loss of memory. Panel B: Effects on memory of varying the retention interval, filled with variable AS ("Monday ... Tuesday ..."). Data are from McFarlane \& Humphreys (2012);

Lewandowsky et al. (2008); Lewandowsky, Geiger, et al. (2010). Extending the disctractor period has clear detrimental effects on performance.

Figure 2. Possible modes of interaction between two modes of rehearsal. (A) The effects of the two rehearsal processes are additive, hence any cause of forgetting ( F) can only be fully counteracted when both modes are operating (top). When one mode is eliminated, for example by articulatory suppression (center), restoration of memory is impaired. In consequence, memory remains stable over an extended retention interval only if both forms of rehearsal can operate. As soon as one of them is blocked, forgetting over time occurs, and when both are blocked, there is more forgetting, as shown with idealized data in the bottom panel. (B). The two types of rehearsal are each fully sufficient on their own, and therefore memory is not impaired when one of them is eliminated (center panel). Only when both are blocked, forgetting over time is observed, as is shown with idealized data in the bottom panel. See text for details.

Figure 3. The exponential growth of encoding strength, $\eta$, as a function of time. Each panel shows 100 samples of rate $r$ drawn from a normal distribution with mean $R=6$ and standard deviation $s=1$, representing 100 randomly different encoding events. One such randomly-selected event is illustrated by the continuous line. The duration of an event, $t$, 
is determined by the time it takes the curve to reach the criterion, which differs between panels: (a) standard encoding event, $\tau=.95$; (b) articulatory rehearsal at $250 \mathrm{~ms} /$ item, $\tau=.78 ;$ (c) attentional refreshing at $50 \mathrm{~ms} /$ item, $\tau=.26$.

Figure 4. Distribution of overt rehearsal patterns observed by Tan \& Ward (2008) during encoding of 6-item lists for immediate serial recall. From top to bottom, the three panels refer to fast ( $1 \mathrm{~s} /$ word $)$, medium $(2.5 \mathrm{~s} /$ word $)$, and slow $(5 \mathrm{~s} /$ word $)$ presentation speed, respectively. See text for explanation of the various rehearsal schedules. A rehearsal set refers to the set of words rehearsed overtly after the presentation of each list item. Figure reprinted from Tan, L \& Ward, G., 2008, Psychonomic Bulletin 85 Review, 15, 535-542, published by Springer, reprinted with permission.

Figure 5. Top panel: Serial position curves observed with overt rehearsal reported by Tan \& Ward (2008). Figure reprinted from Tan, L \& Ward, G., 2008, Psychonomic Bulletin \& Review, 15, 535-542, published by Springer, reprinted with permission. Bottom panel: Most plausible serial position curves obtained in Simulation 1 (decay rate 0.3, rehearsal duration $0.5 \mathrm{~s})$.

Figure 6. Distribution of rehearsal regimes observed in Simulation 1 with two different rehearsal speeds, $0.25 \mathrm{~s}$ (left panel), and $0.50 \mathrm{~s}$ (right panel). From top to bottom, the three rows of panels refer to fast $(1 \mathrm{~s} /$ word $)$, medium $(2.5 \mathrm{~s} /$ word $)$, and slow $(5 \mathrm{~s} /$ word $)$ list presentation speeds, respectively. Unfilled bars represent fixed rehearsals, gray bars represent partial cumulative rehearsal, and black bars represent cumulative rehearsal. Any rehearsal orders not falling into the other classes are cross-hatched.

Figure 7. Results of Simulation 1. From top to bottom, the three rows of panels refer to fast $(1 \mathrm{~s} /$ word $)$, medium $(2.5 \mathrm{~s} /$ word $)$, and slow $(5 \mathrm{~s} /$ word $)$ presentation speeds, respectively. From left to right, columns of panels refer to recall performance in the 
absence of rehearsal, or with rehearsal durations of $0.25 \mathrm{~s}$ and $0.5 \mathrm{~s}$, respectively. Within each panel, increasing shading of plotting symbols reflects increasing decay rates, which range from 0.2 (unfilled circles) to 0.8 (solid squares).

Figure 8. Results of Simulation 2. Presentation parameters were identical to Simulation 1, and each list was followed by immediate serial recall. The top row of panels shows the

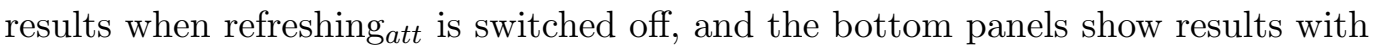
refreshing ${ }_{a t t}$ present. The right-hand panels show the results with rehearsal ${ }_{\text {art }}$ present, whereas the left-hand panels show the results when there was no articulatory rehearsal. Within each panel, the parameters represent the three different presentation speeds.

Figure 9. Results of Simulation 3 involving the complex-span paradigm with a $1 \mathrm{~s} /$ item presentation rate. The top row of panels shows the results when refreshing ${ }_{a t t}$ is switched off, and the bottom panels show results with refreshing att $_{\text {present. The right-hand panels }}$ show the results with rehearsal ${ }_{\text {art }}$ present, whereas the left-hand panels show the results when there was no articulatory rehearsal. Within each panel, high cognitive load (CL) was .625 and low CL was .33.

Figure 10. Results of Simulation 3 averaged across serial positions and shown as a function of the type of rehearsal in operation. Presentation rate was $1 \mathrm{~s} /$ item. High cognitive load (CL) involved a $0.3 \mathrm{~s}$ free-time period after each distractor and low CL involved $1.0 \mathrm{~s}$ free time. Refreshing ${ }_{a t t}$, when present, required $50 \mathrm{~ms} /$ item whereas rehearsal $l_{\text {art }}$ required $0.25 \mathrm{~s} /$ item.

Figure 11. Results of Simulation 4 involving serial recall after a short $(2 \mathrm{~s})$ and long $(4 \mathrm{~s})$ delay after list presentation. List presentation was sufficiently rapid to prevent rehearsal during encoding. Rehearsal during the retention interval proceeded in strict cumulative forward order. The top row of panels shows the results when refreshing ${ }_{a t t}$ is switched off, 
and the bottom panels show results with refreshing att $_{\text {present. The right-hand panels }}$ show the results with rehearsal ${ }_{\text {art }}$ present, whereas the left-hand panels show the results when there was no articulatory rehearsal.

Figure 12. Effects of articulatory rehearsal on strengthening of two list items. Shading of circles and superimposed numbers refers to the extent of activation of each item or context element (on an arbitrary scale), and thickness of lines indicates strength of association weights between an item and its context markers. Panel A shows the state of memory before rehearsal commences. Both items are associated to their overlapping context markers. Panel B: First item is cued for rehearsal by activating the first context marker. Item 1 is most active and is hence retrieved for rehearsal. Panel C: Item 1 is re-encoded and the context-to-item associations are strengthened (by a factor of 3 in this example). Panel D: The second item is cued for rehearsal but Item 1 is more active because of its recent rehearsal.

Figure 13. Distribution of rehearsal regimes observed with SOB-CS in Simulation 5 with a rehearsal speed of $0.25 \mathrm{~s}$ per item. From top to bottom, the three rows of panels refer to fast ( $1 \mathrm{~s} /$ word), medium $(2.5 \mathrm{~s} /$ word), and slow $(5 \mathrm{~s} /$ word) list presentation speeds, respectively. Unfilled bars represent fixed rehearsals, gray bars represent partial cumulative rehearsal, and black bars represent cumulative rehearsal. Any rehearsal orders not falling into the other classes are cross-hatched.

Figure 14. Serial position curves obtained by SOB-CS in Simulation 5. The solid lines represent predictions with maintenance rehearsal for the three different presentation rates used by Tan \& Ward (2008). The dashed line shows predictions when rehearsal is prevented and interference from articulatory suppression is introduced. 
Figure 15. Serial position curves obtained with elaborative rehearsal in SOB-CS in Simulation 6, using three different presentation rates. Elaborative rehearsal is instantiated by increasing the distinctiveness of rehearsed items. 
Rehearsal and Short-Term Retention, Figure 1

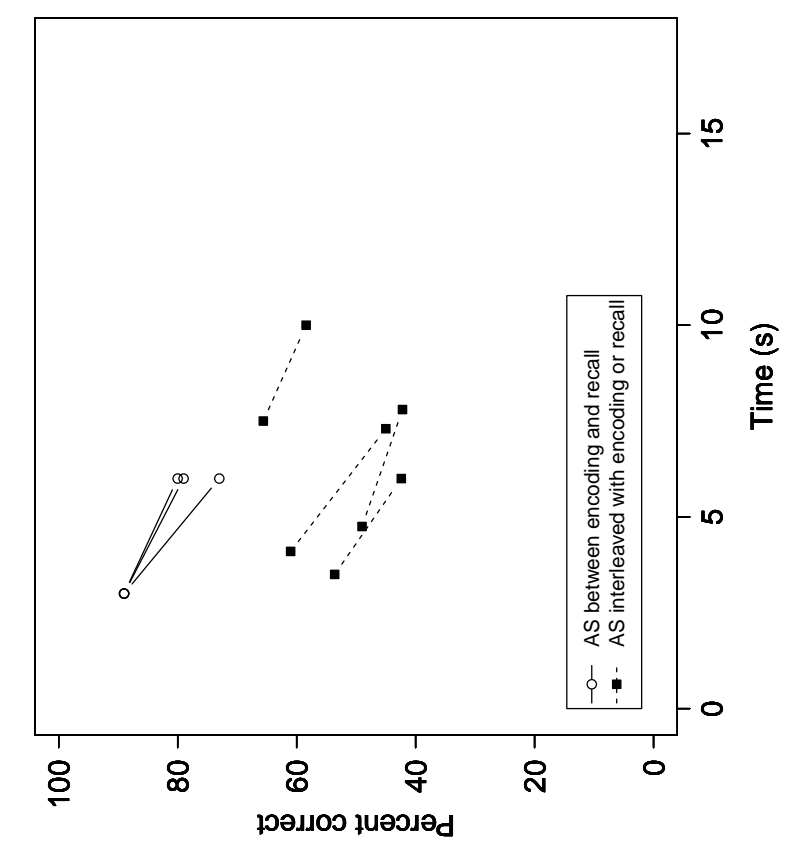

อ

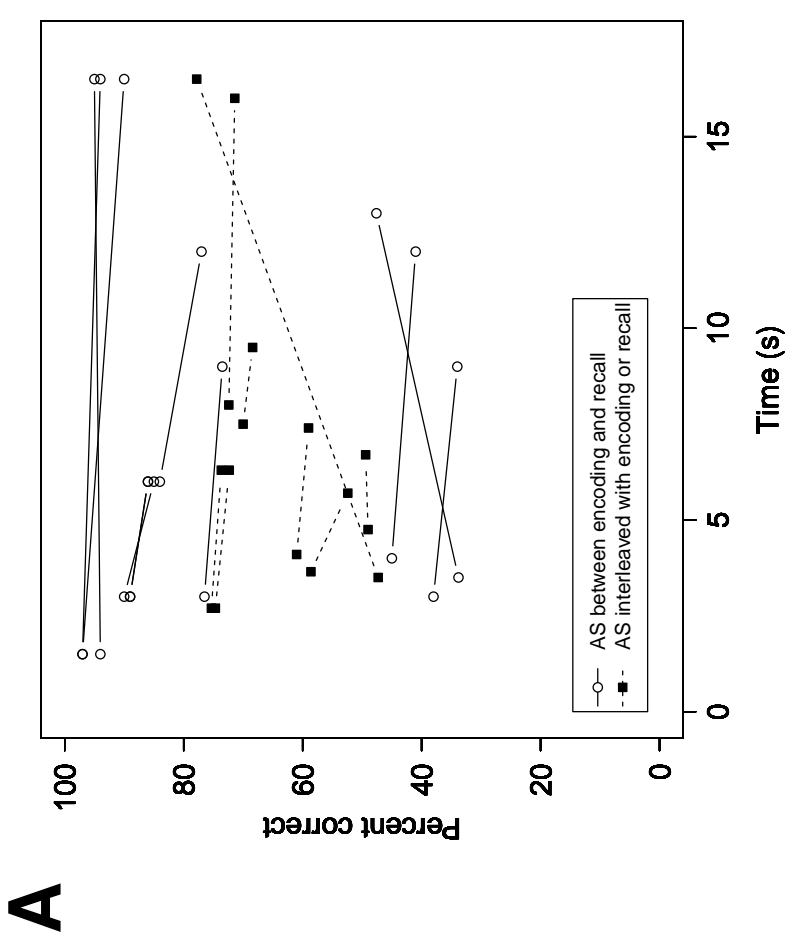


Rehearsal and Short-Term Retention, Figure 2

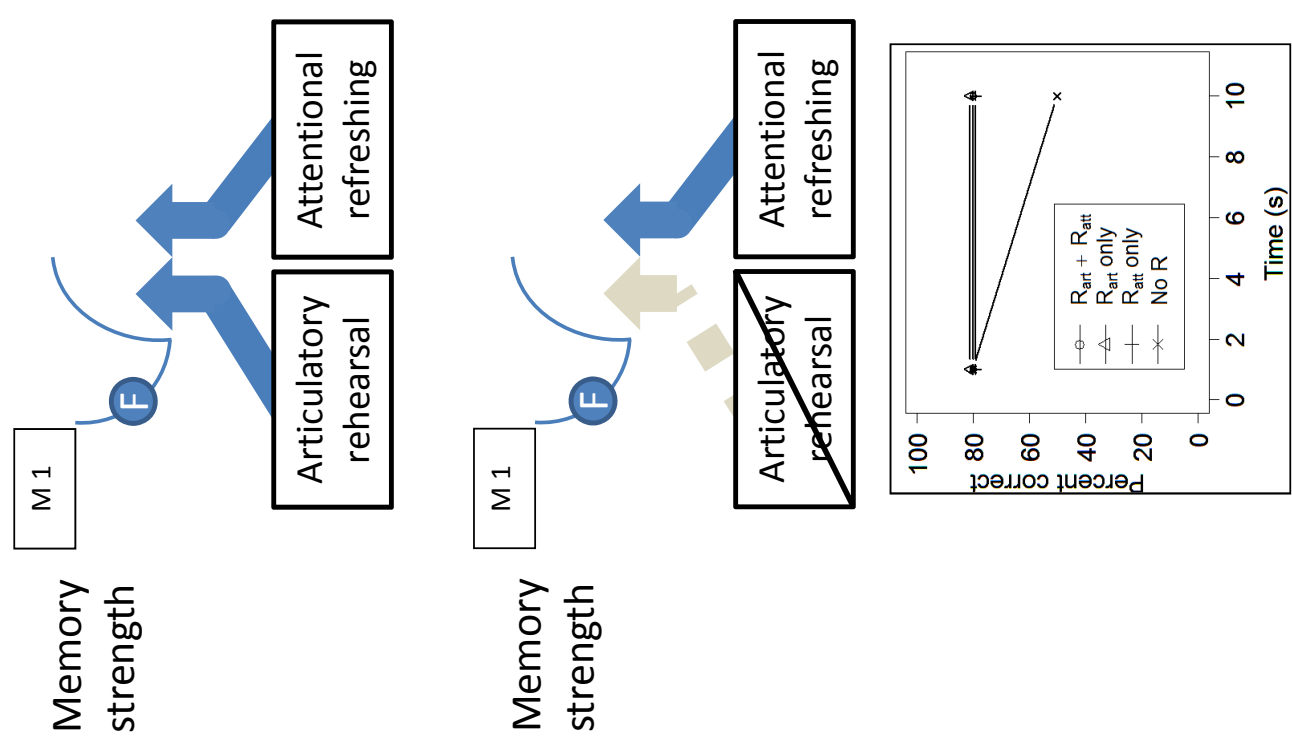

\section{ค}
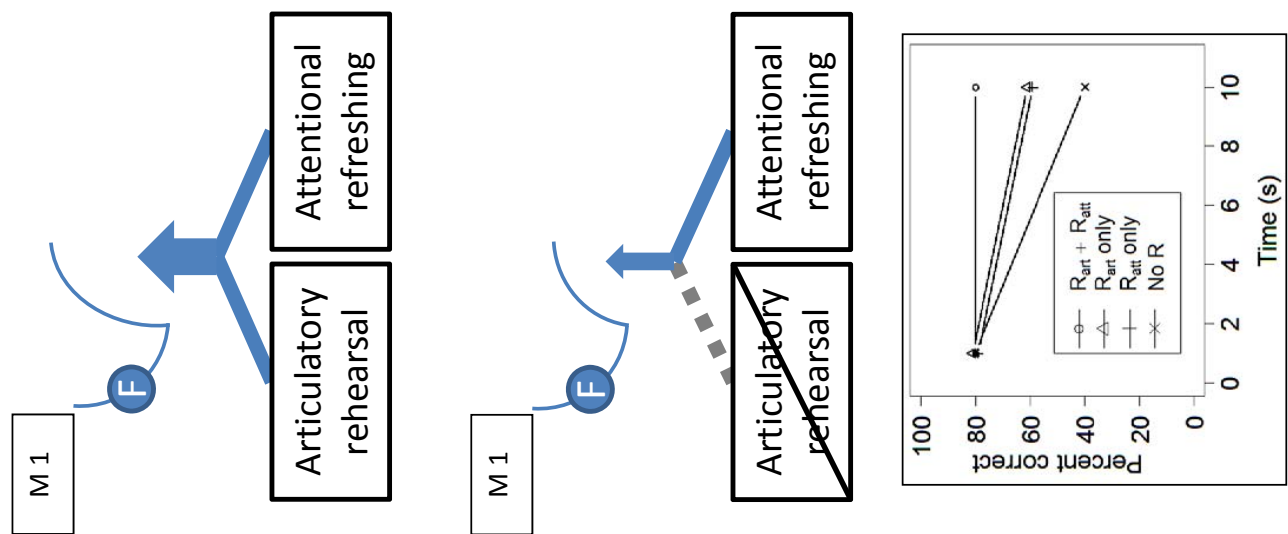

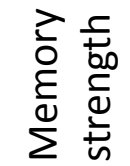

곧담

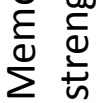

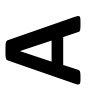


Rehearsal and Short-Term Retention, Figure 3

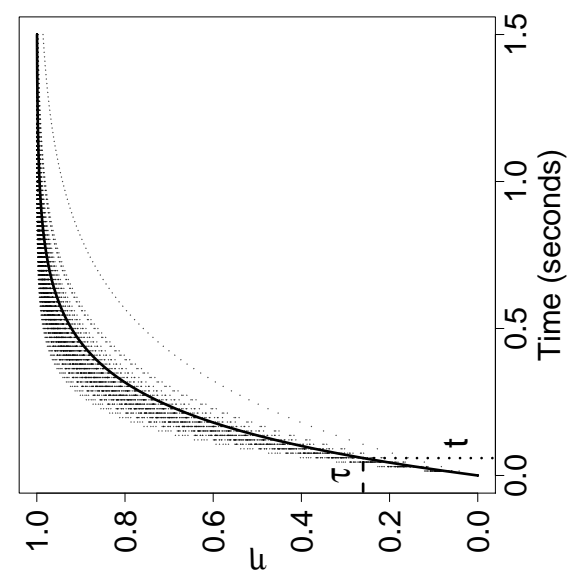

0

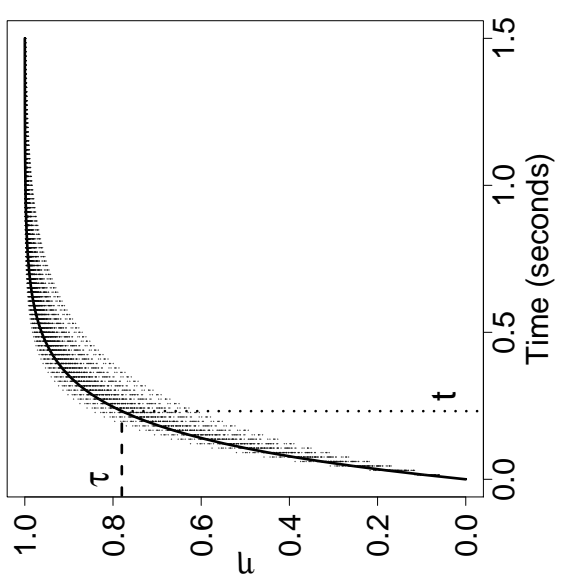

ด

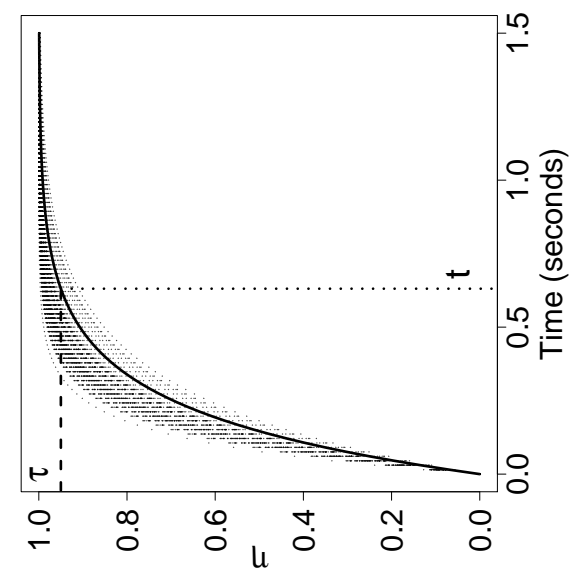


Fast Presentation Rate: Proportion of Rehearsal Strategies Used in Each Rehearsal Set

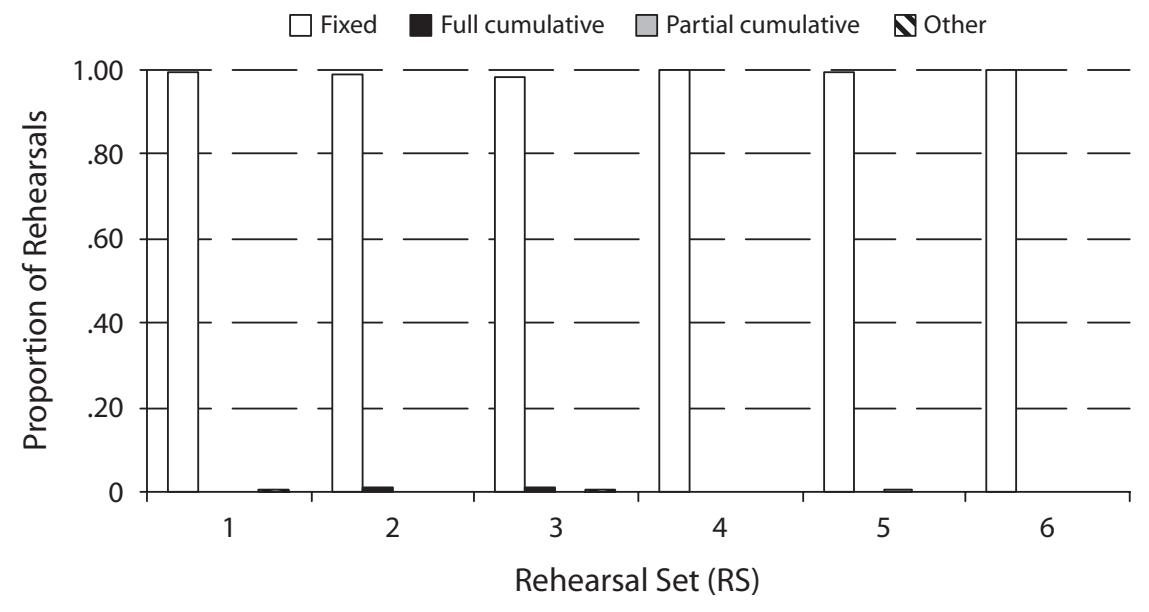

Medium Presentation Rate: Proportion of Rehearsal Strategies Used in Each Rehearsal Set

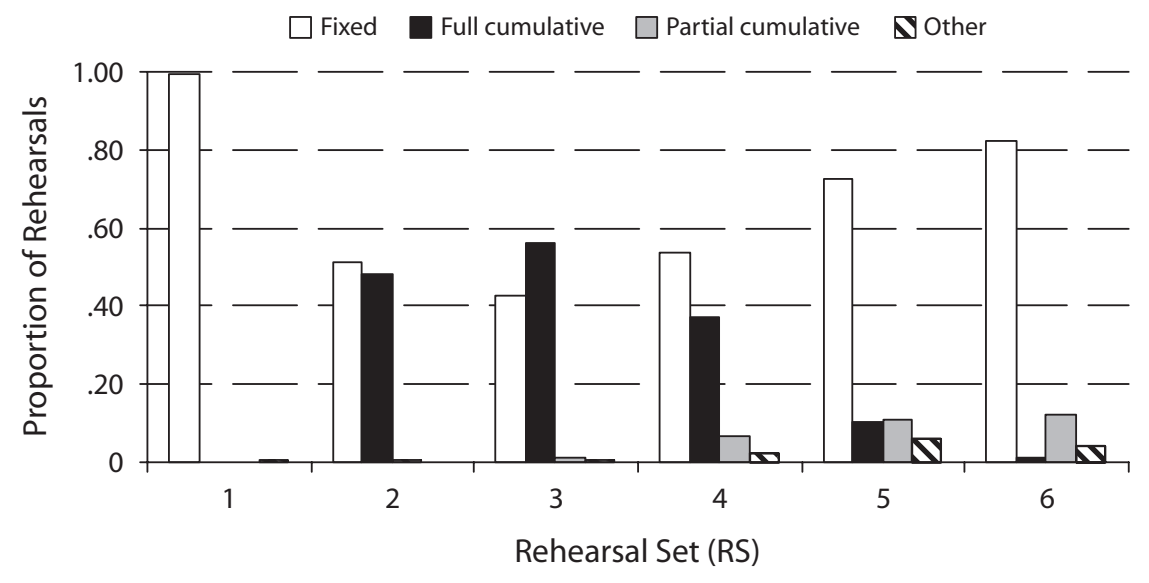

Slow Presentation Rate: Proportion of Rehearsal Strategies Used in Each Rehearsal Set

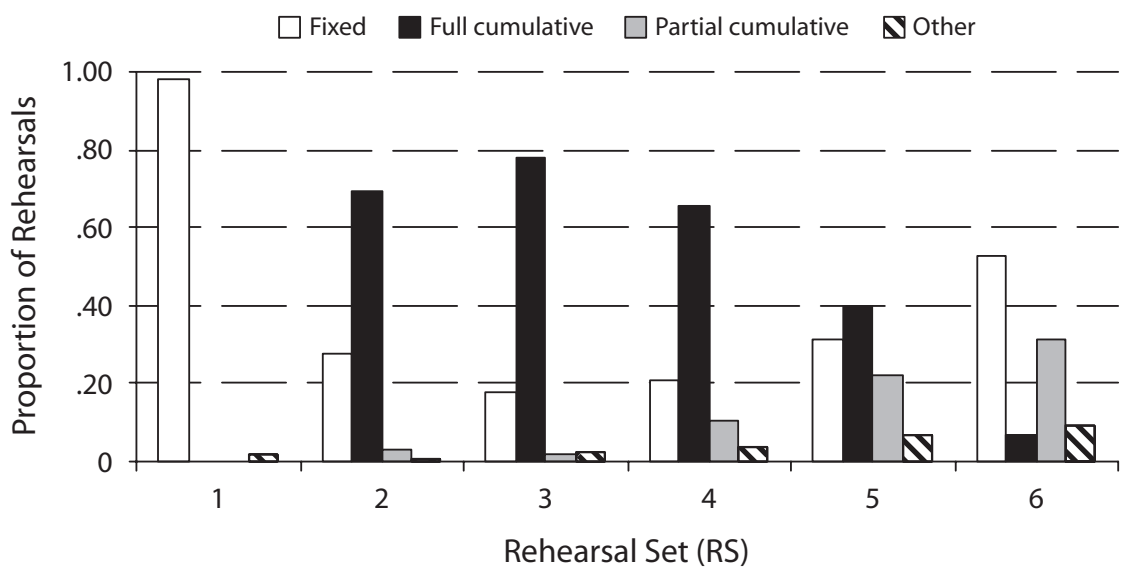


Rehearsal and Short-Term Retention, Figure 5
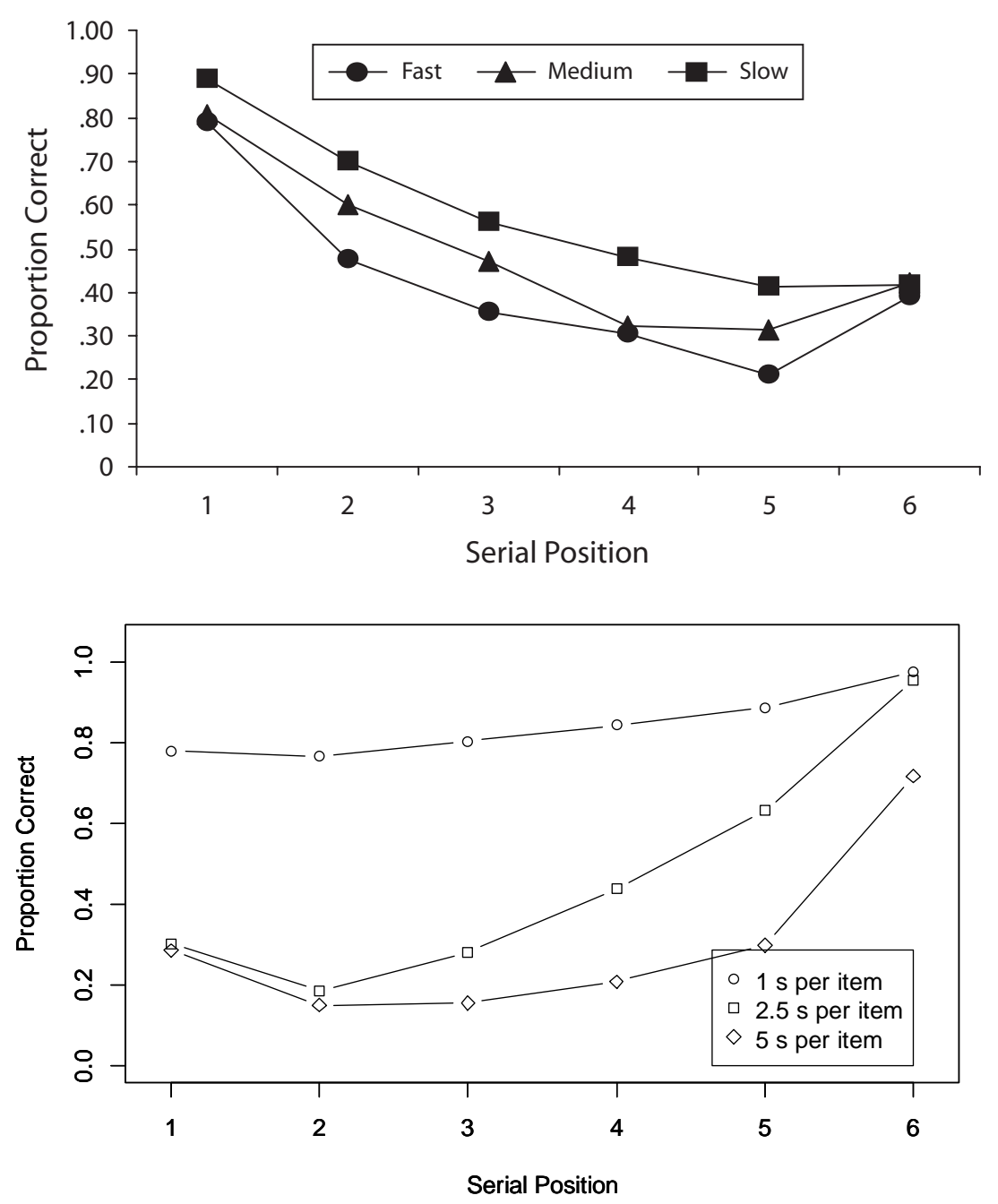
Rehearsal and Short-Term Retention, Figure 6
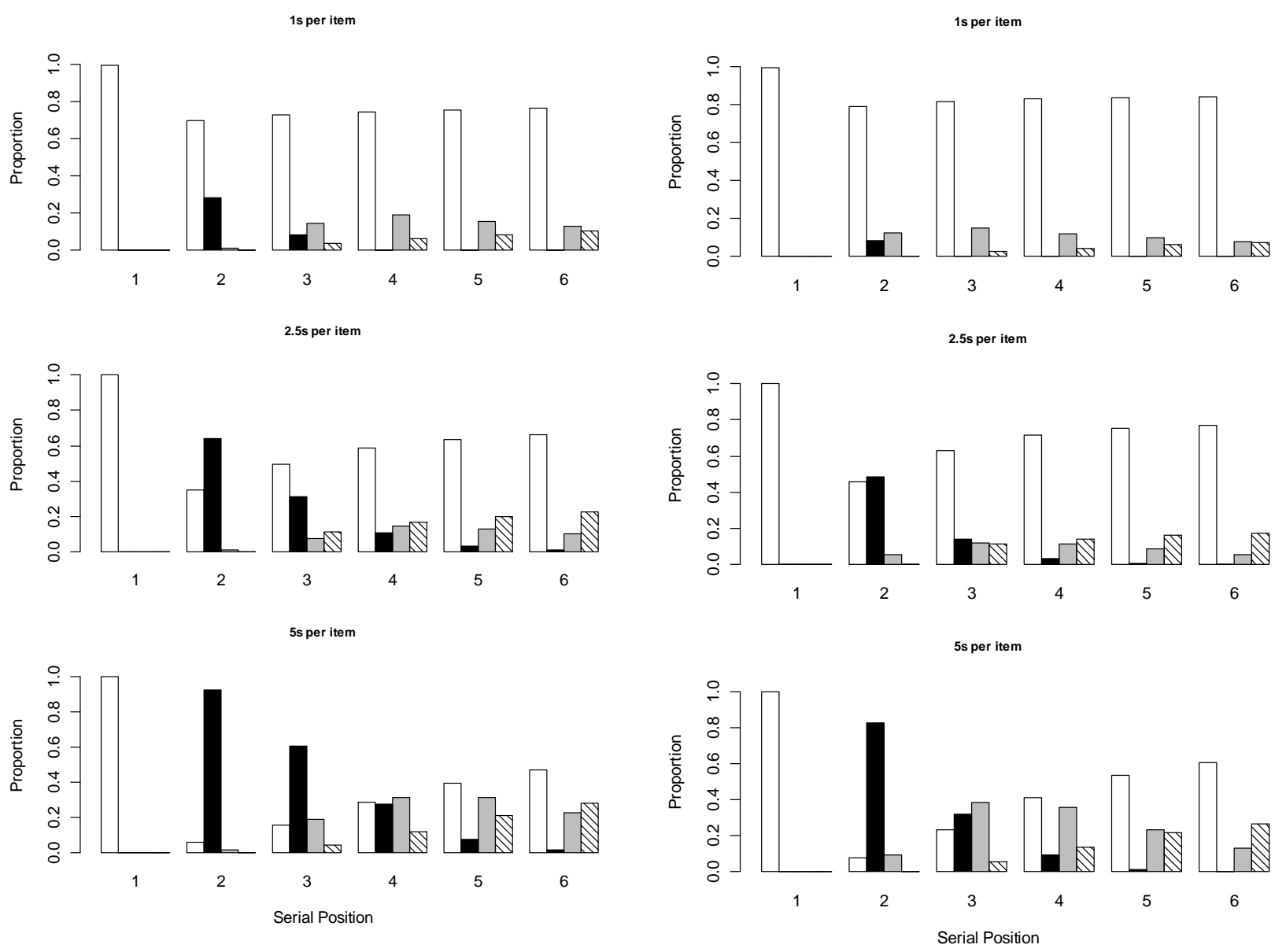
Rehearsal and Short-Term Retention, Figure 7
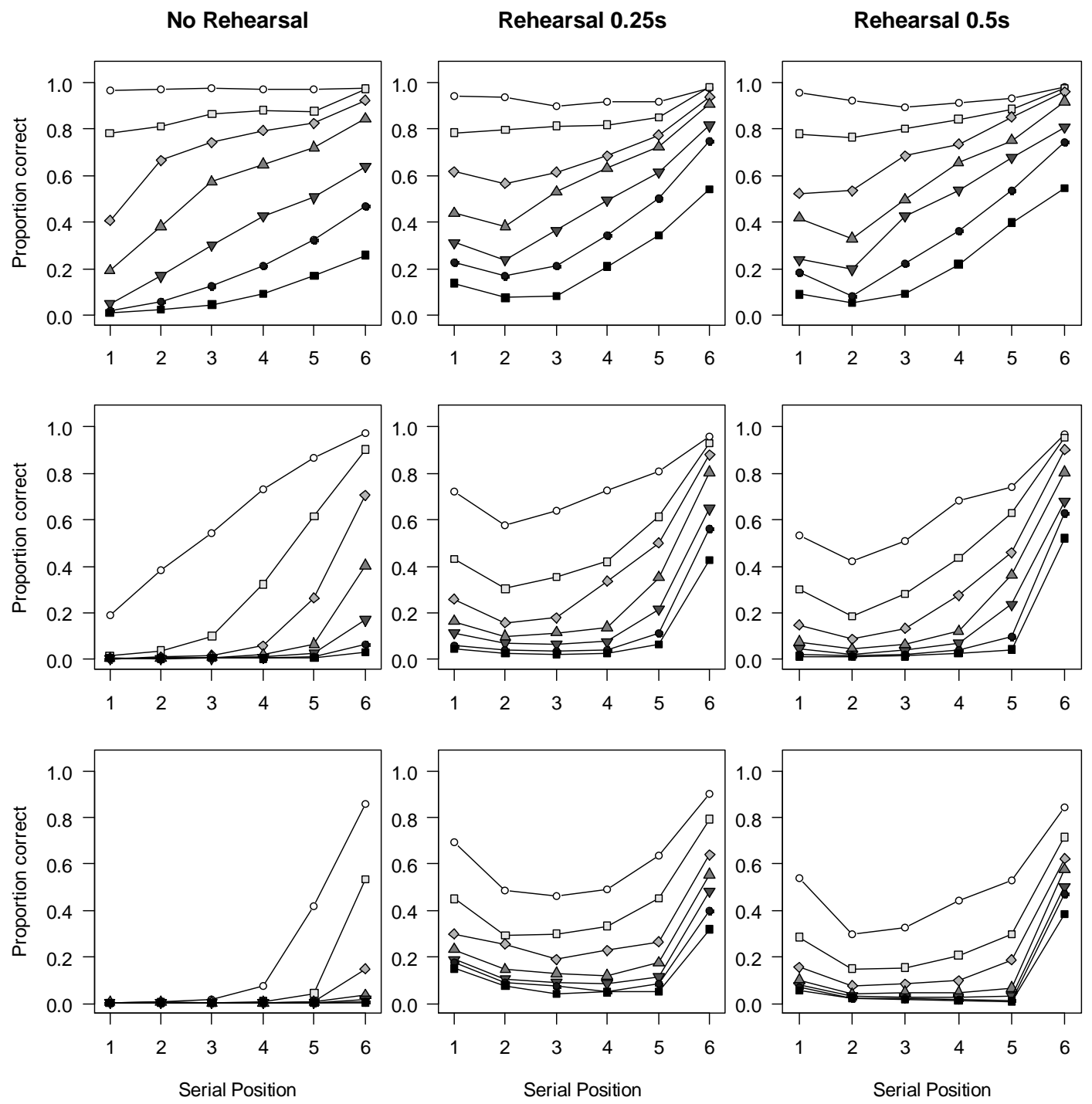
Rehearsal and Short-Term Retention, Figure 8
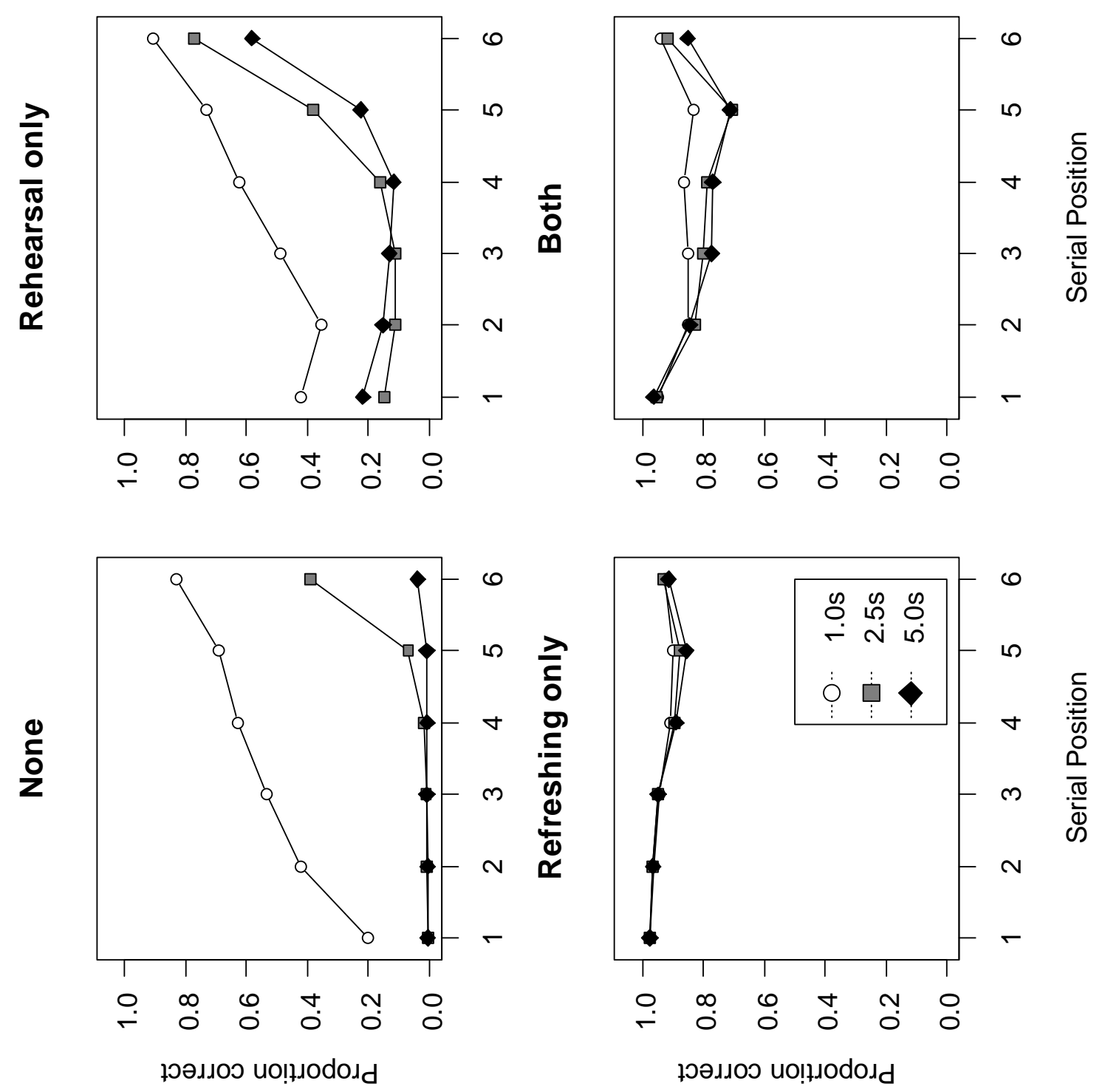
Rehearsal and Short-Term Retention, Figure 9
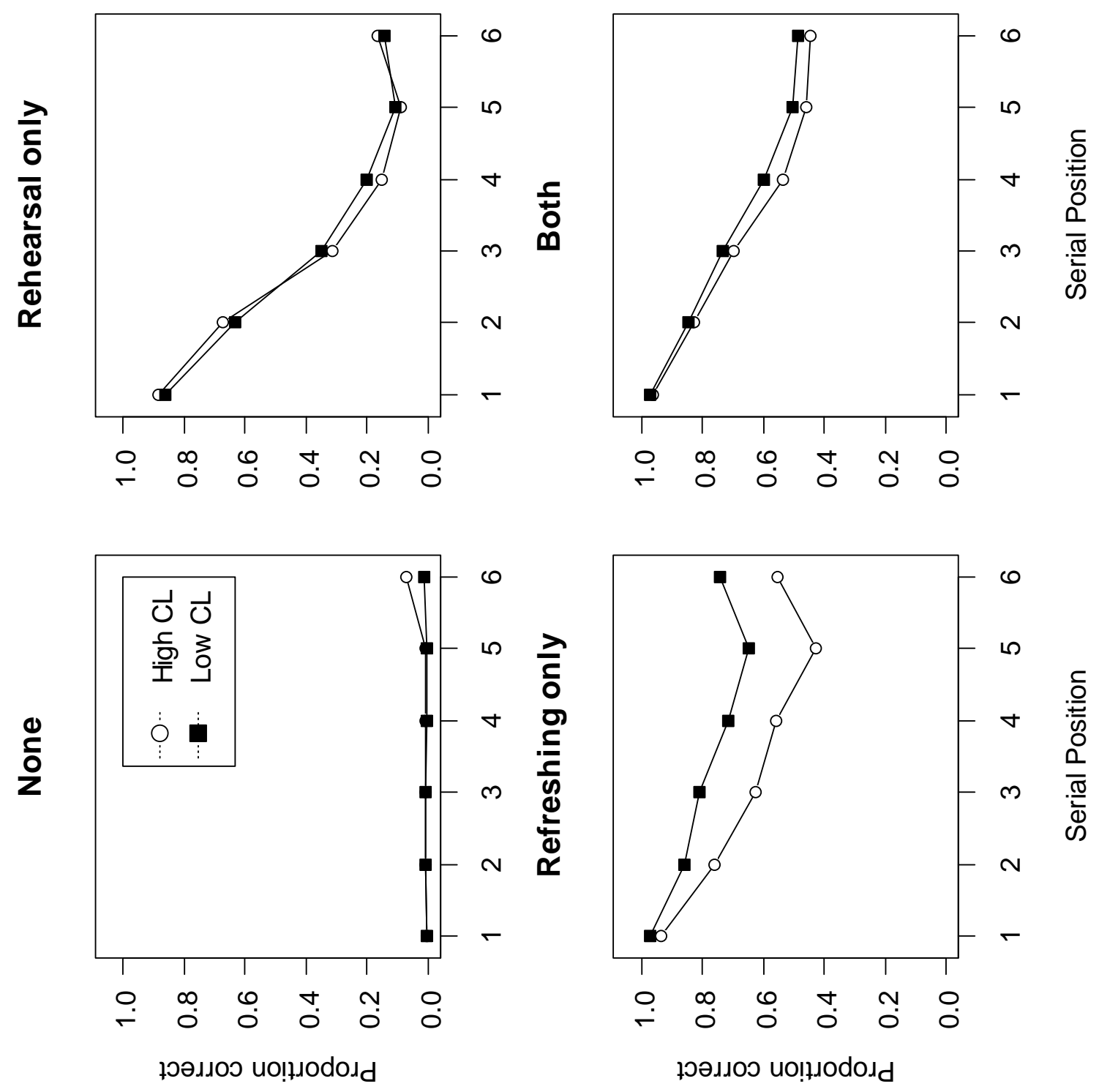
Rehearsal and Short-Term Retention, Figure 10

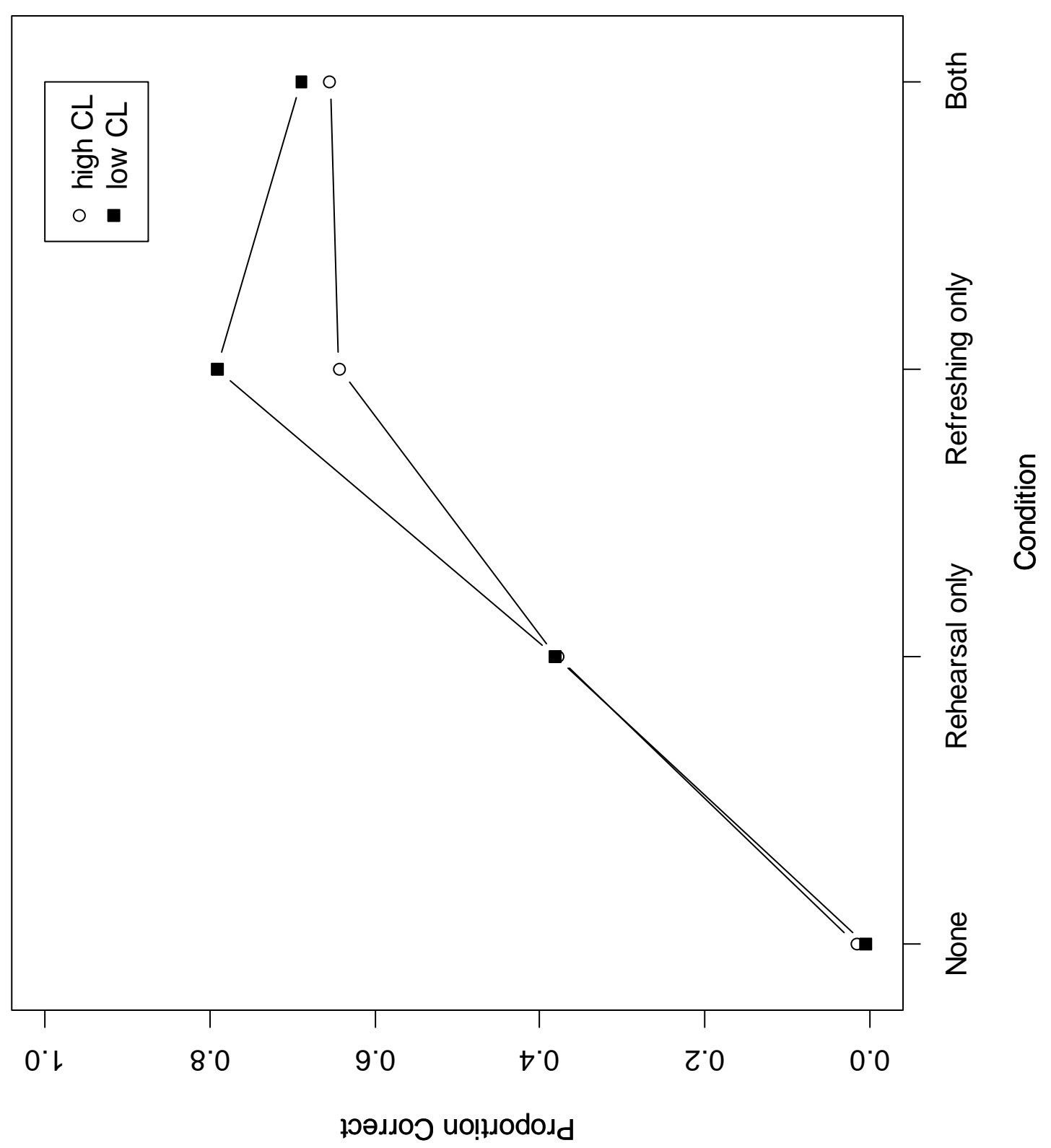


Rehearsal and Short-Term Retention, Figure 11
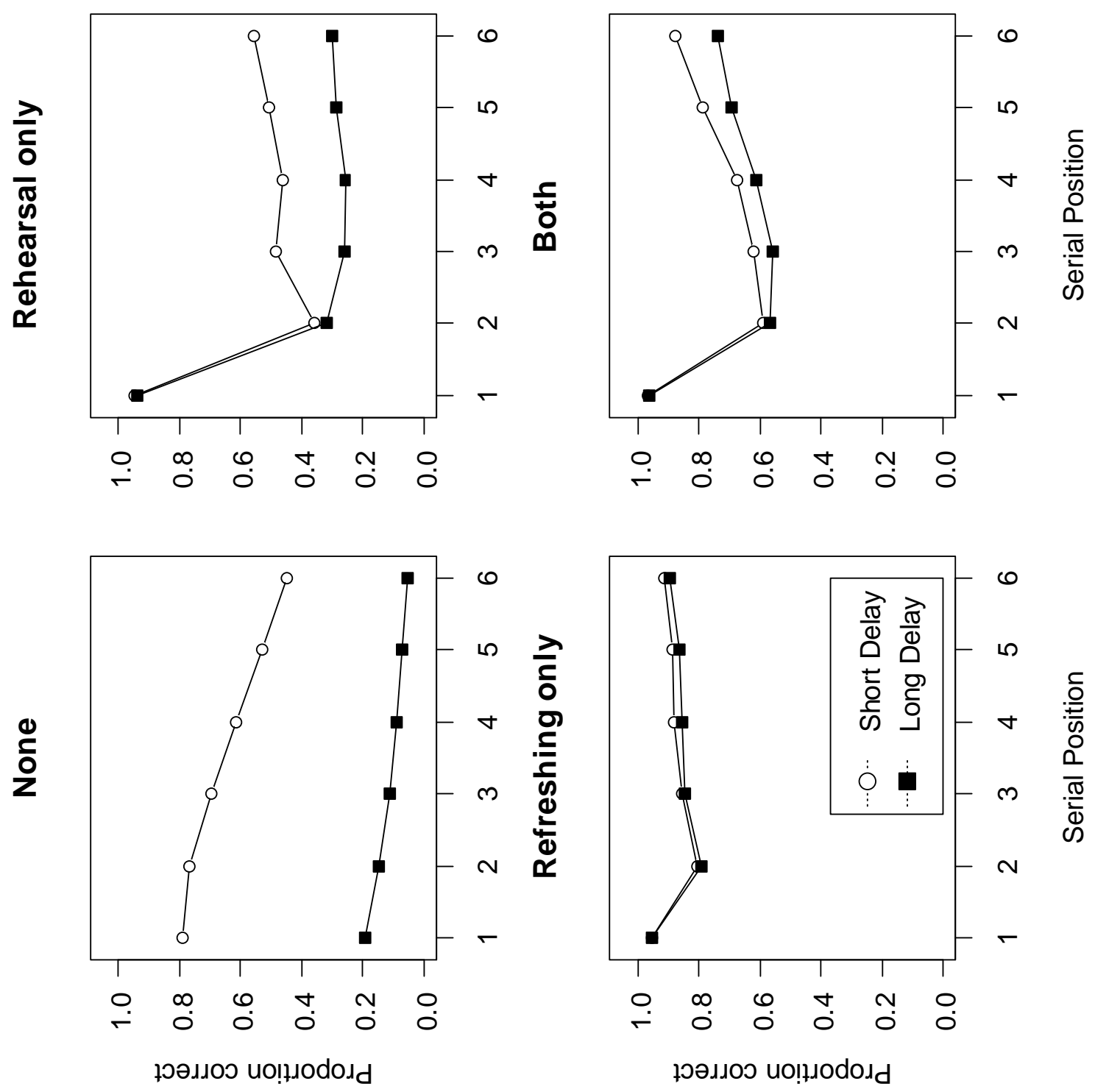
Rehearsal and Short-Term Retention, Figure 12

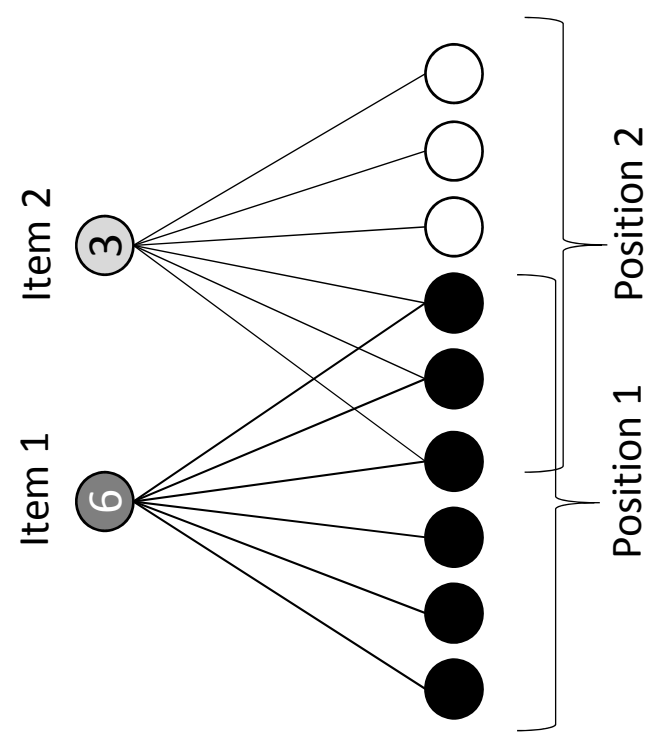

$\infty$
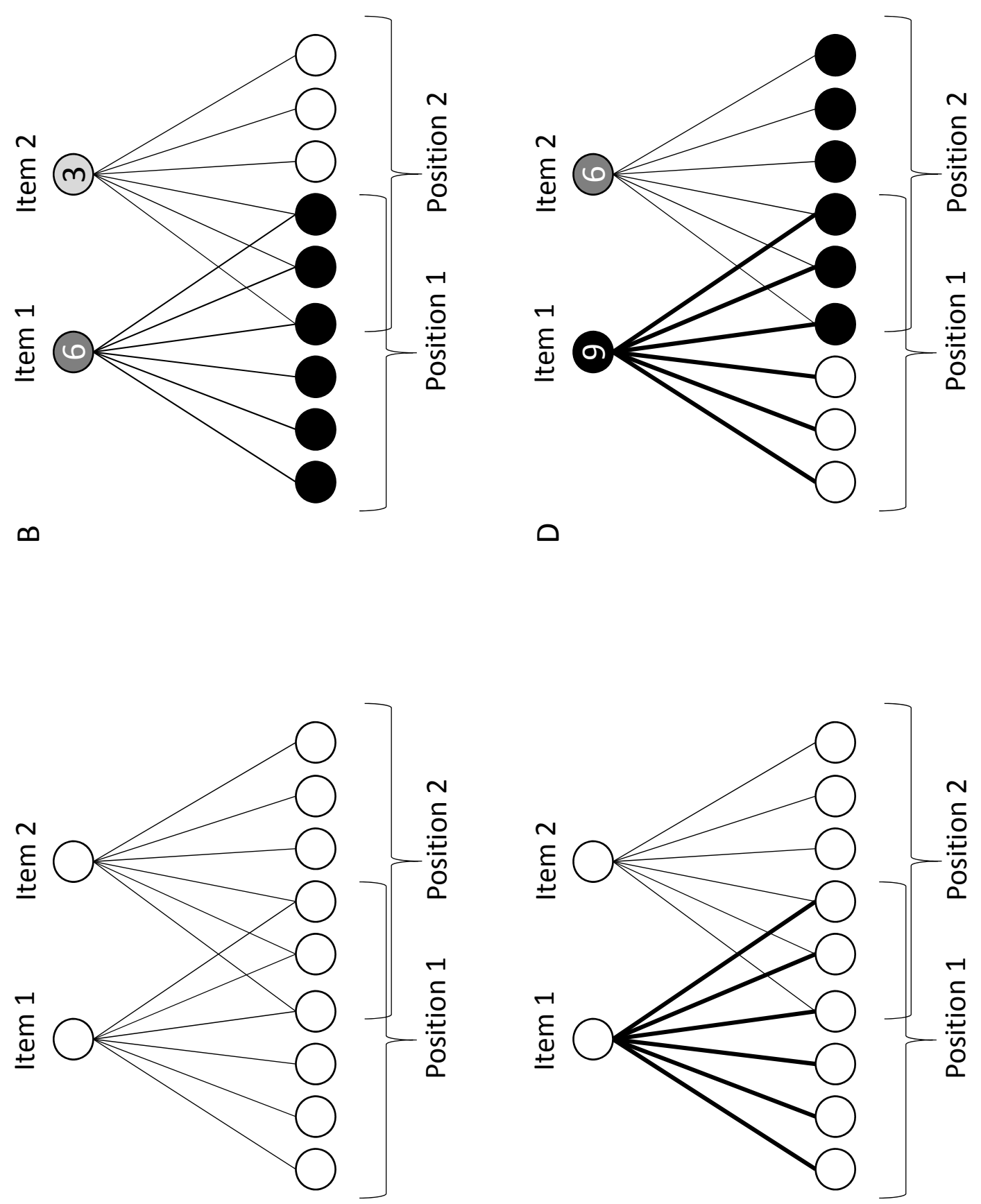

$\varangle$

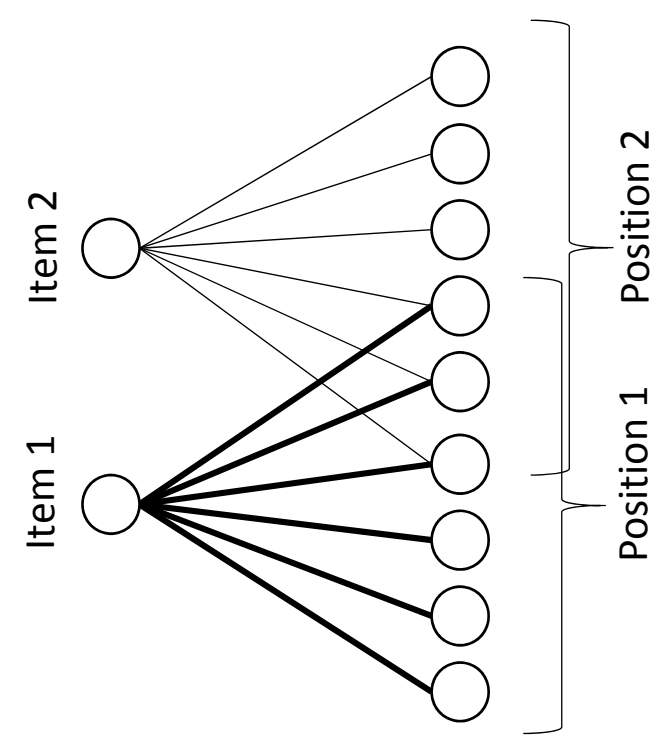

$\cup$ 
Rehearsal and Short-Term Retention, Figure 13
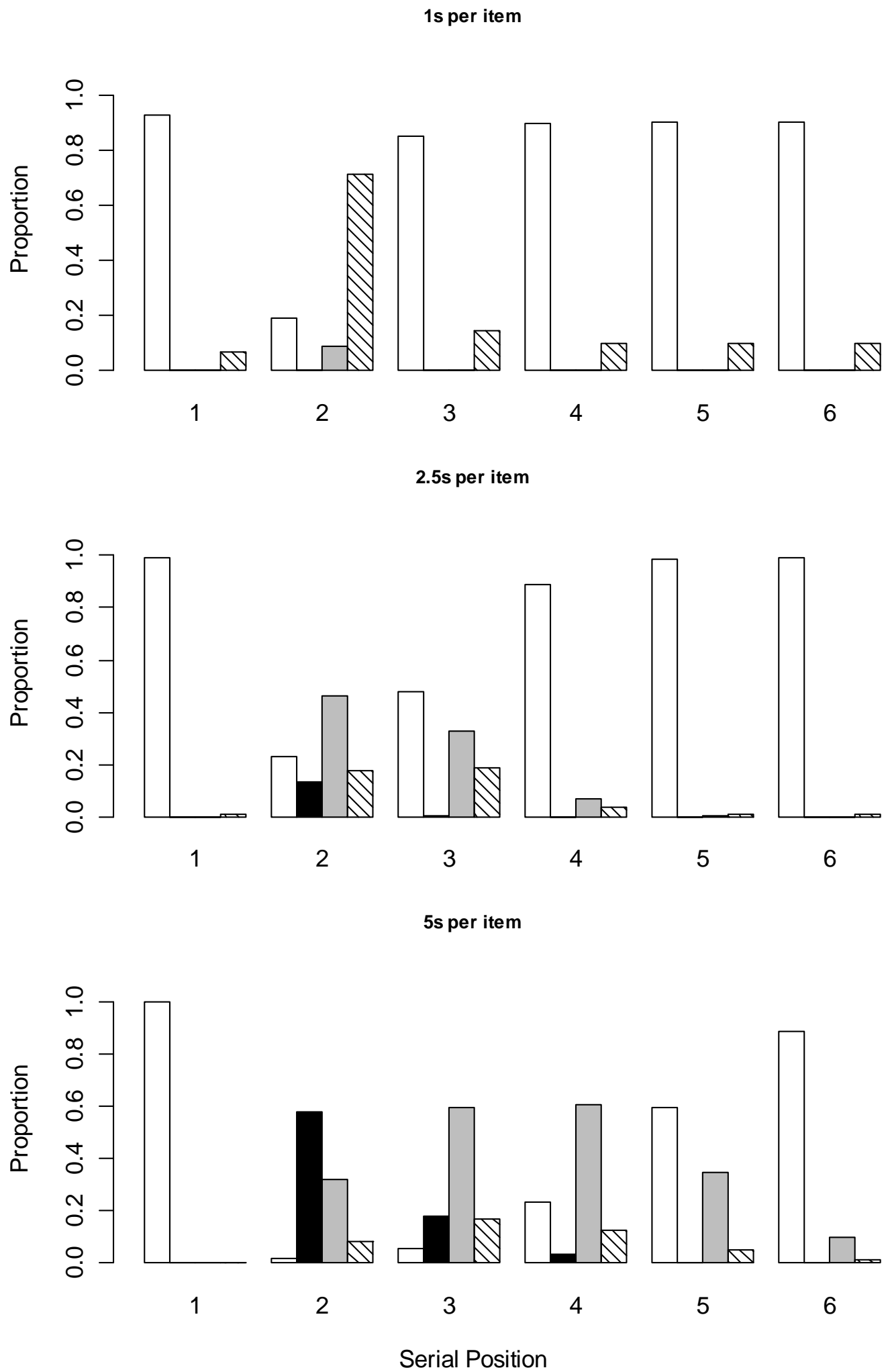
Rehearsal and Short-Term Retention, Figure 14

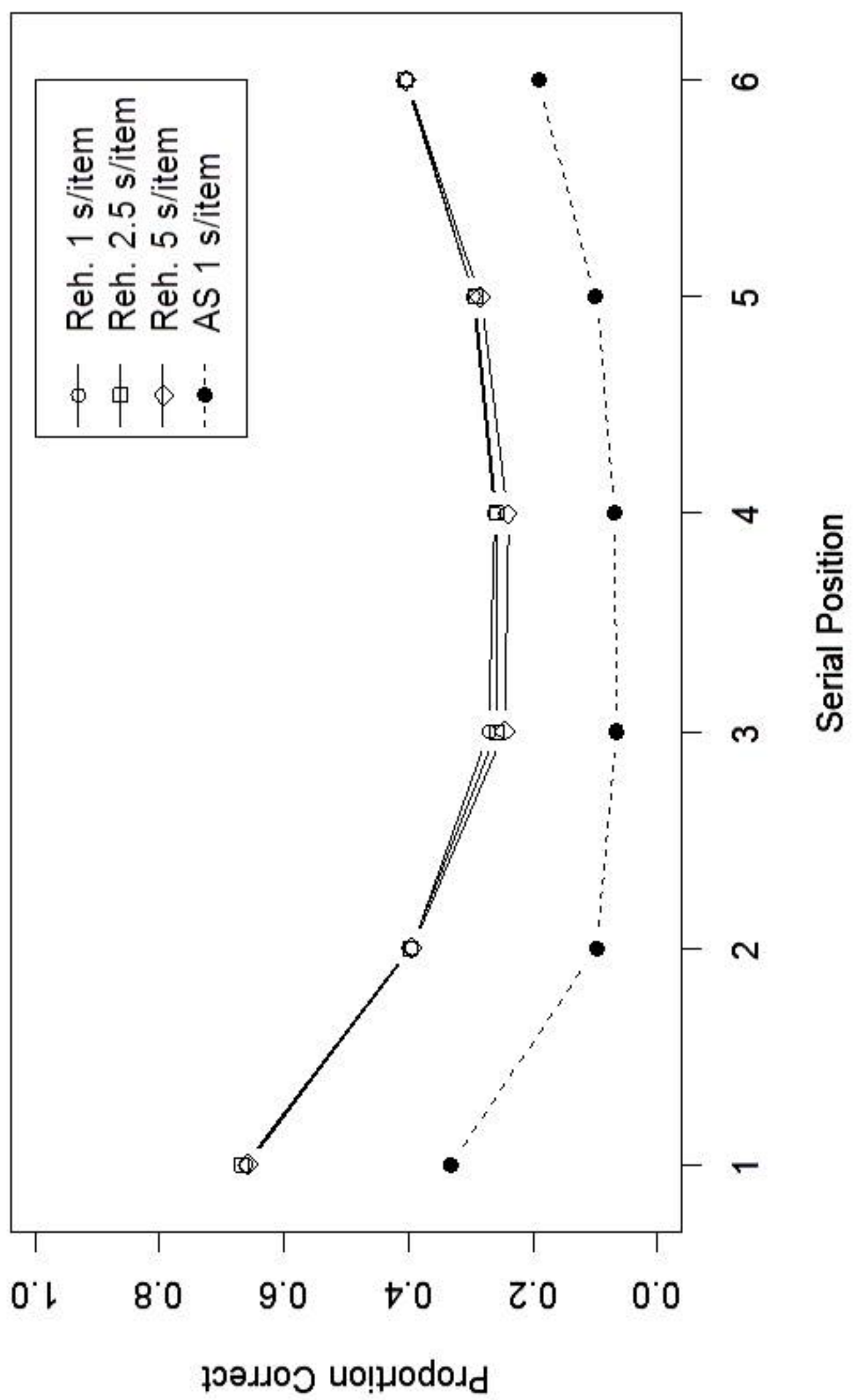


Rehearsal and Short-Term Retention, Figure 15

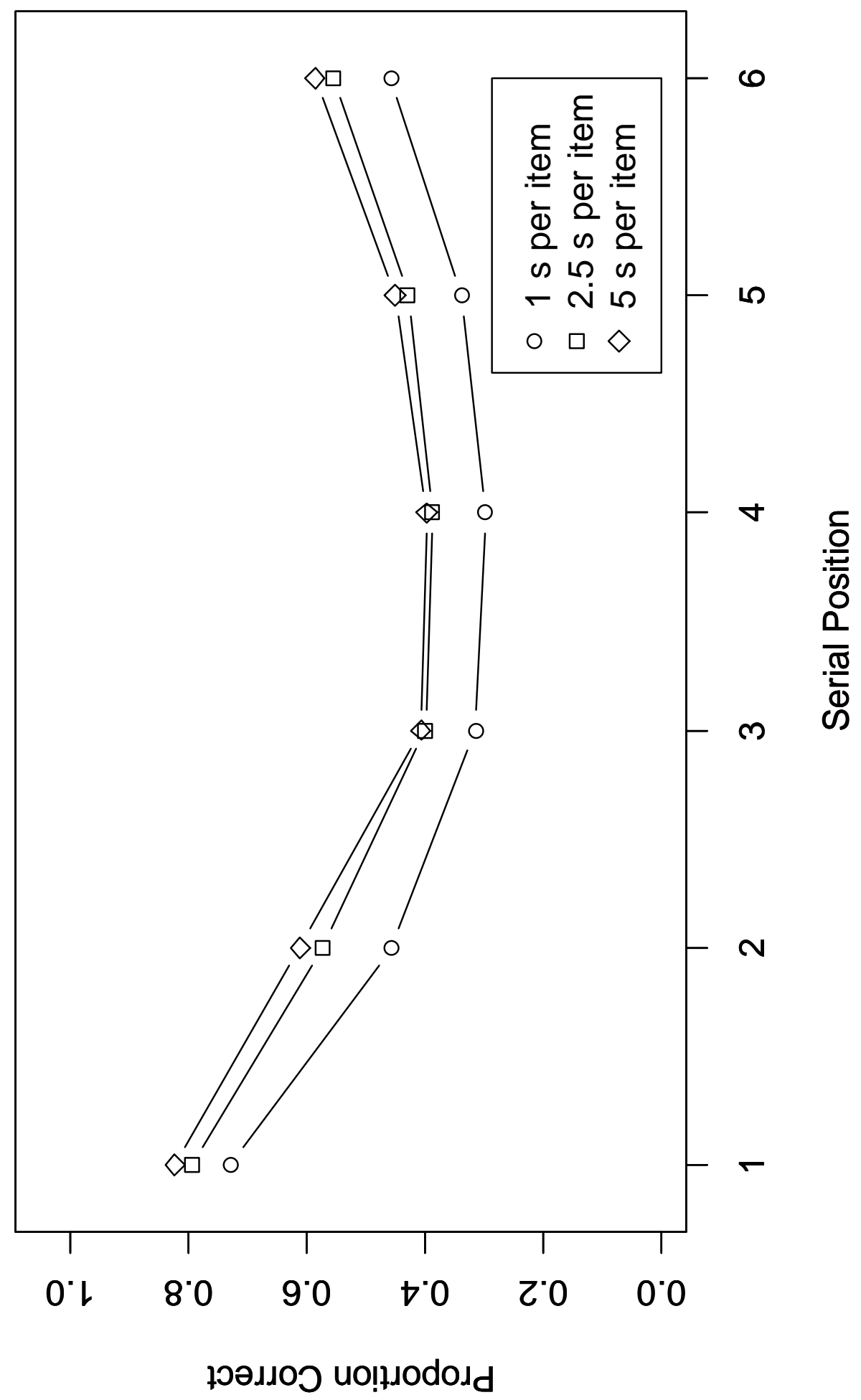

